\title{
Comparative proteomic analysis for revealing the advantage mechanisms of salt-tolerant tomato (Solanum lycoperscium)
}

\author{
Qiang Wang ${ }^{1,2}{ }^{2}$ Baike Wang ${ }^{2}$, Huifang Liu ${ }^{2}$, Hongwei Han ${ }^{2}$, Hongmei Zhuang ${ }^{2}$, Juan Wang ${ }^{2}$, Tao Yang ${ }^{2}$, Hao \\ Wang ${ }^{\text {Corresp., } 2}$, Yong Qin ${ }^{\text {Corresp. } 1}$ \\ ${ }^{1}$ College of Horticulture $\square$ Xinjiang Agricultural University, Urumqi, China \\ 2 Institute of Horticulture Crops, Xinjiang Academy of Agricultural Sciences, Urumqi, China \\ Corresponding Authors: Hao Wang, Yong Qin \\ Email address: wanghao183@163.com, xjndqinyong@126.com
}

Salt stress causes the quality change and significant yield loss of tomato. However, the resources of salt-resistant tomato were still deficient and the mechanisms of tomato resistance to salt stress were still unclear. In this study, the proteomic profiles of two salttolerant and salt-sensitive tomato cultivars were investigated to decipher the saltresistance mechanism of tomato and provide novel resources for tomato breeding. We found high abundance proteins related to nitrate and amino acids metabolismsin the salttolerant cultivars. The significant increase in abundanceof proteins involved in Brassinolides and GABA biosynthesis were verified in salt-tolerant cultivars, strengthening the salt resistance of tomato. Meanwhile, salt-tolerant cultivars with higher abundance and activity of antioxidant-related proteins have more advantages in dealing with reactive oxygen species caused by salt stress. Moreover, the salt-tolerant cultivars had higher photosynthetic activity based on overexpression of proteins functioned in chloroplast, guaranteeing the sufficient nutrient for plant growth under salt stress. Furthermore, three key proteins were identified as important salt-resistant resources for breeding salt-tolerant cultivars, including sterol side chain reductase, gamma aminobutyrate transaminase and starch synthase. Our results provided series valuable strategies for salt-tolerant cultivars which can be used in future. 


\section{Comparative proteomic analysis for revealing the advantage mechanisms of}

\section{Salt-tolerant tomato (Solanum lycopersicum)}

3 Qiang Wang 1,2, Baike Wang ${ }^{2}$, Huifang Liu ${ }^{2}$, Hongwei Han ${ }^{2}$, Hongmei Zhuang ${ }^{2}$, Juan Wang ${ }^{2}$,

4 Tao Yang ${ }^{2}$, Hao Wang ${ }^{2 *}$, Yong Qin ${ }^{1 *}$

5

$6 \quad{ }^{1}$ College of Horticulture, Xinjiang Agricultural University, Urumqi 830052,China ;

$7{ }^{2}$ Institute of Horticulture Crops, Xinjiang Academy of Agricultural Sciences, Urumqi 830091, China;

8

$9 *$ Corresponding author: Hao Wang, E-mail: wanghao183@163.com

$10 *$ Corresponding author:Yong Qin, E-mail: xjndqinyong@126.com

11

12

13

14

15

16

17

18

19

20

21

22

23

24

25

26

PeerJ reviewing PDF | (2021:06:62808:2:0:NEW 19 Jan 2022) 


\section{ABSTRACT}

29 Salt stress causes the quality change and significant yield loss of tomato. However, the resources of salt-resistant tomato were still deficient and the mechanisms of tomato resistance to salt stress

31 were still unclear. In this study, the proteomic profiles of two salt-tolerant and salt-sensitive 32 tomato cultivars were investigated to decipher the salt-resistance mechanism of tomato and 33 provide novel resources for tomato breeding. We found high abundance proteins related to 34 nitrate and amino acids metabolisms in the salt-tolerant cultivars. The significant increase in 35 abundance of proteins involved in Brassinolides and GABA biosynthesis were verified in salt36 tolerant cultivars, strengthening the salt resistance of tomato. Meanwhile, salt-tolerant cultivars

37 with higher abundance and activity of antioxidant-related proteins have more advantages in 38 dealing with reactive oxygen species caused by salt stress. Moreover, the salt-tolerant cultivars 39 had higher photosynthetic activity based on overexpression of proteins functioned in chloroplast, 40 guaranteeing the sufficient nutrient for plant growth under salt stress. Furthermore, three key 41 proteins were identified as important salt-resistant resources for breeding salt-tolerant cultivars, 42 including sterol side chain reductase, gamma aminobutyrate transaminase and starch synthase. 43 Our results provided series valuable strategies for salt-tolerant cultivars which can be used in 44 future.

Keywords: Tomato (Solanum lycopersicum); Salt-tolerant; Proteome; Salt tolerant variety 
INTRODUCTION

57 Soil salinization is a worldwide problem affecting agricultural production. With the destruction 58 of soil caused by the expansion of the global industrial scale, nearly $20 \%$ of cultivated land and 59 nearly half of the irrigated land are threatened by salt damage to varying degrees in the world. 60 Soil salinization has become a major environmental problem which has been widely realized 61 (Rozema and Flowers 2008). The increasing trend of salinization poses a great threat to the 62 sustainable development of agriculture. Currently, the agricultural improvement measures of 63 salinization soil mainly include rational fertilization, increasing organic fertilizer and restraining 64 soil salt super accumulation(Chen, 2011).However, the cultivation of saline-tolerant crop 65 varieties is still an important method, which is meaningful to the improvement and utilization of 66 salinized soil. As an important cash crop in the world, tomato growing was limited by various 67 factors relevant to salt stress, resulting in a loss of yield. However, the salt-tolerance mechanism 68 of tomato is still unclear and need further in-depth researches.

The major harmful ions in saline and alkaline soils is $\mathrm{Na}^{+}$. The accumulation of $\mathrm{Na}^{+}$in soils could result in osmotic stress (Xiong \& Zhu, 2002; Munns\& Tester, 2008), ion toxicity (Tester \& Davenport, 2003) and oxidative stress (Hasegawa et al., 2000; Xiong \& Zhu, 2002), which profoundly influence the growth and development of plants. The excessive accumulation of $\mathrm{Na}^{+}$ in plant cells results in the destruction of the dynamic balance between the generation and clearance of oxygen free radicals, and leads to or intensifies the membrane lipid peroxidation and membrane lipid defatination (Senaratna et al.,1985), which is harmful to the normal physiological progresses of plants. Additionally, excessive $\mathrm{Na}^{+}$decreases the concentration of

$77 \mathrm{Ca}^{2+}$ in the plasma membrane, resulting in membrane structure destruction and changes in 78 function, and intracellular exosmosis of potassium, phosphorus and organic solute (Tuna et al., 2007), which inhibited plant growth and development. It has been proved that salt stress can influence the utilization rate of starch, protein, fatty acids and other macromolecular substances 
81 in seeds, resulting in seed germination failure or delayed germination (Lovato et al.,1994;

82 Ungar,1996). Meanwhile, the repair and reconstruction of cell membrane system of seeds were

83 also impaired under saline-alkali stress. The membrane ion selective absorption capacity is

84 decreased under salt stress, accompanying with ion toxicity and generation of free radicals which

85 further damage the seed colloidal structure and slow down the development of seeds

86 (Almansouri et al.,2001; Chartzoulakis and Loupanaki 1997). Furthermore, salt stress could

87 significantly influence the structure of plasma membrane. Salt stress can also lead the formation

88 of large vacuoles, local expansion of endoplasmic reticulum, decrease in chloroplast numbers

89 and enlargement of mitochondria (Mitsuya et al., 2000; Bruns and Hecht-Buchholz 1990).

90 Currently, various mechanisms of plant tolerance to salt stress have been elucidated, including

91 reprogramming the photosynthetic pathway (Allakhverdiev et al.,2002; Mittal et al., 2012; Wang

92 et al.,2021), enhancing the antioxidant protection ability (Gupta et al.,1993;Parvin et

93 al.,2019;Faizan et al.,2021) and synthesis and accumulation of osmotic regulating substances

94 (Yang et al., 2007; Muhammad et al., 2021). It has been reported that salt stress induce the

95 increase in activity of SOD and APX, which plays important roles in the antioxidant protection

96 system of plants (Zhang and Mu 1993). Compared with wild-type tobacco, salt-resistant cultivars

97 have higher photosynthetic efficiency and are more resistant to salt stress (Gupta et al., 1993).

98 Under salt stress, plants usually need to synthesize and accumulate a large amount of solutes

99 with active and non-toxic osmotic effects to reduce water potential (Chen, 2011), allowing cells

100 to absorb water in a hypotonic environment. Moreover, plants also activate multiple signaling

101 pathways to resist the harms caused by salt stress, including salt oversensitive (SOS) signal

102 transduction pathway, jasmonic acid, mitogen-activated protein kinase (MAPK), and abscisic

103 acid (ABA) signaling pathways (Xiong et al., 2002; Zhu, 2002; Colcombet\& Hirt, 2008).

104 Therefore, plants regulate salt tolerance through a complex network of resistance response.

105 Tomato is a worldwide vegetable crop whose tolerance mechanism and proteomics of salt stress

106 have been investigated extensively and deeply (Manaa, A., et al.,2013; Gong et al.,2014). 
107 Comparative proteomic analyses are highly effective in the identification of protein changes that

108 are associated with genotypic properties. For instance, an iTRAQ analysis of proteomes from

109 Maize inbred lines at salt-tolerant genotype F63 and the salt-sensitive genotype F35 showed that

110 a large number of proteins was affected by salt in the two accessions (Cui et al.,2015). Those

111 differentially expressed proteins may be helpful for further elucidating salt tolerance mechanisms.

112 The salt-tolerant introgression line IL7-5-5 was constructed by using 76 wild tomato Solanum

113 Pennellii LA716 as donor and cultivated tomato M82 as acceptor. The salt-tolerant line IL7-5-5

114 was screened by repeated salt stress method at seedling stage. breeders observed that IL7-5-5

115 plants were salt-tolerant while M82 plants were susceptible to salt stress. In this study, we found

116 that various proteins relevant to salt resistance were highly expressed in salt-tolerant cultivars,

117 resulting in series advantages of ST in permeability regulation, maintaining normal

118 photosynthesis and removing reactive oxygen species produced by salt stress. Our research

119 provides a new blueprint for breeding salt tolerance tomato.

120 MATERIALS AND METHODS

\section{Plant material and Experimental design}

122 The experiment was carried out in the sunlight greenhouse of Xinjiang Academy of Agricultural

123 Sciences in April 2019. The seeds of salt-tolerant infiltration system IL7-5-5 (ST) and salt-

124 sensitive M82 (SS) were seeded in peat, perlite and vermiculite (volume ratio 1:1:1) substrates,

125 seedling raising in conventional greenhouse. The roots were cleaned when the seedlings grow to

126 the four-true-leaf stage, then transplanted the plant into 1/2 concentration Hoagland standard

127 nutrient solution for hydroponic culture. The seedlings were cultivated at $25 / 18^{\circ} \mathrm{C}$ (day/night)

128 with a relative humidity of $40 \%$, and at a photoperiod of $14 \mathrm{~h} / 10 \mathrm{~h}$ (day/night). The oxygen was

129 supplied to culture chambers three times everyday and 30 minutes every time. The evaporated

130 water was replenished to its initial volume every two days, this culture method last for one week

131 and the treatment started after this.The following experimental groups were arranged with 3

132 replicates and treated as follows:(1)CK: 1/2 Hoagland Nutrient Solution; (2) Salt Stress

133 Treatment:1/2 Hoagland nutrient solution with $200 \mathrm{mmol} \cdot \mathrm{L}^{-1} \mathrm{NaCl}$. Sample the location of the 
134 same part of the material to be tested at 0,1 , and $12 \mathrm{~h}$ after treatment. The leaves were selected

135 and stored in the refrigerator at $-80{ }^{\circ} \mathrm{C}$. All sample collection and preparation procedures were

136 performed in triplicate, and each independent biological experiment has 3 technological

137 replicates. Data were exhibited as the means \pm standard deviations (SDs).

\section{Determination of Physiological indicators}

139 Tomato leaves at $0 \mathrm{~h}$ and $1 \mathrm{~h}$ after salt treatment were used for Superoxide dismutase (SOD) 140 activity, Polyamine oxidase (PAO) activity, Peroxidase (POD) activity, Polyphenol oxidase

141 (PPO) activity, Soluble sugar content and $\mathrm{H}_{2} \mathrm{O}_{2}$ content assays by physiological trait kits (Suzhou

142 Comin Biotechnology Co. Suzhou, China) according to the manufacturer's instructions. A 143 completely randomized block design was used, and all experiments had three replicates.

144 Experimental data were analyzed by two-way analysis of variance (ANOVA) and Tukey $(P<$

145 0.05) comparisons were used to analyze differences.

\section{Protein extraction, Trypsin digestion and Isobaric Labeling}

147 Frozen tissue from tomato leaves was ground in liquid nitrogen using a mortar and pestle. Dry 148 samples were mixed with lysis buffer (1\% TritonX-100, $10 \mathrm{mM}$ dithiothreitol, 1\% protease 149 inhibitor cocktail and $2 \mathrm{mM}$ EDTA) and then processed three times on ice using a high intensity 150 ultrasonic processor. After centrifugation $\left(12,000 \mathrm{~g}, 4^{\circ} \mathrm{C}, 10 \mathrm{~min}\right)$ using Tris-saturated phenol 151 ( $\mathrm{pH} \mathrm{8.0)}$ and transferring the supernatant, add at least 4 volumes of ammonium sulfate saturated

152 methanol and allow the protein to precipitate overnight at $20^{\circ} \mathrm{C}$. Remove the supernatant by 153 centrifugation $\left(4^{\circ} \mathrm{C}, 10 \mathrm{~min}\right)$ and wash three times with ice-cold acetone. Protein concentration 154 was determined using the BCA kit. Protein was precipitated at $20 \%(\mathrm{~m} / \mathrm{v})$ TCA. Protein 155 precipitates recovered after vortex mixing incubation $\left(4^{\circ} \mathrm{C}, 2 \mathrm{~h}\right)$ and centrifugation $\left(4500 \mathrm{~g}, 4^{\circ} \mathrm{C}\right.$, $1565 \mathrm{~min}$ ) were washed with pre-chilled acetone, and the dried protein samples were re-dissolved in 157 200nM TEAB and digested overnight with trypsin $(1: 50 \mathrm{w} / \mathrm{w})$. Samples were reduced with 5 $158 \mathrm{mM}$ dithiothreitol $\left(37^{\circ} \mathrm{C}, 60 \mathrm{~min}\right)$ and alkylated with $11 \mathrm{mM}$ iodoacetamide (room temperature,

$15945 \mathrm{~min}$ ) in the dark. Peptide Isobaric labeling was performed using a TMT kit (Thermo Fisher 160 Scientific, Waltham, MA, USA), with SS samples labeled with TMT tags 126, 127 and 128, and 
161 ST samples using TMT tags 129, 130 and 131.

\section{Protein Identification by LC-MS/MS}

163 Separation of samples into fractions by high $\mathrm{pH}$ reversed-phase HPLC using Agilent 300 Extend

164 C18 column (5 $\mu$ m particles, $4.6 \mathrm{~mm}$ I.D., $250 \mathrm{~mm}$ length). The tryptic peptides were dissolved

165 in solvent $\mathrm{A}(0.1 \%$ formic acid, $2 \%$ acetonitrile in water), then loading all the solution into a 166 home-made reversed-phase analytical column. The whole operation was conducted on an EASY-

167 nLC 1000 Ultra Performance Liquid Chromatography system with a constant flow rate of 400

$168 \mathrm{nl} / \mathrm{min}$, and the detail information was followed: the gradient was comprised of an increase from

$1696 \%$ to $23 \%$ solvent in $98 \%$ acetonitrile over $26 \mathrm{~min}, 23 \%$ to $35 \%$ with 8 min and then climbing

170 to $80 \%$ in $3 \mathrm{~min}$, finally, hold at $80 \%$ for $3 \mathrm{~min}$. The information of instrument applied during

171 experiment was followed: electrospray voltage of $2.0 \mathrm{kv}, \mathrm{m} / \mathrm{z}$ scan range of 350 to 1800 for full

172 scan, and intact peptides detecting resolution of 70,000. NCE was used to select peptides and the

173 Orbitrap with a resolution of 17,500 was used to detect the fragments.

\section{Data analysis}

175 Maxquant search engine (vs 1.6.3.3, Cox and Mann, 2008) and uniprot database was use to

176 process MS/MS data. Several protein types should be classified for accurate analysis and the

177 detail information was followed: carbamidomethyl on Cys was recongnized as fixed 178 modification and acetylation on protein N-terminal or oxidation on Met and deamidation (NQ)

179 were classified as variable modifications. TMT-6plex quantification has also been performed.

180 More software operation parameters were followed: mass tolerance for fragment ions was 0.02

181 Da, first search of mass tolerance for precursor ions was $20 \mathrm{ppm}$, main search of mass tolerance 182 for precursor ions was 5 ppm, FDR was adjusted to $<1 \%$ and minimum score for modified 183 peptides was set $>40$.

\section{Enrichment Analysis}

185 Gene ontology and KEGG pathway enrichment analyses were carried out by R packages cluster 186 profiler. Proteins were classified by GO annotation into three categories: biological process, 187 cellular compartment and molecular function. For each GO category, a two-tailed Fisher's exact 
188 test was employed to test the enrichment of the differentially expressed protein against all 189 identified proteins. Both pathway and GO terms with a p-value $<0.05$ is considered significant.

190 Furthermore, wolfpsort were used for a subcellular localization predication to predict the protein

191 subcellular localization.

192 Protein-protein Interaction Network

193 All candidate proteins were searched against the STRING database version 10.1 for protein-

194 protein interactions. Only interactions between the proteins belonging to the searched data set

195 were selected, thereby excluding external candidates. Interaction network form STRING was

196 visualized in Cytoscape 3.0. The settings used in STRING database was shown as below:

197 Network type: full STRING network ( the edges indicate both functional and physical protein

198 associations ); meaning of network edges: evidence; active interaction sources: Textmining、

199 Experiments、Databases、Co-expression、Neighborhood、Gene Fusion and Co-occurrence.

200 qRT-PCR verification of gene expression of autophagy-related proteins

201 The expression of important salt related proteins (A0A0C6G3Q8, A0A3Q71RF5 and

202 A0A37J2V8) was investigated by real-time quantitative polymerase chain reactions (RT-qPCR).

203 Primer 6(v6.24) Designer was used to design the RT-qPCR specific primers (Supplementary

204 Table1). The method of total RNA extraction, quantification and cDNA synthesis were

205 described previously. Vieira et al(2016). All experiments have been carried out at least three

206 times with three independent biological replicates. The relative expression levels of target genes

207 was calculated by the method of fold change of $2^{-\triangle \Delta \mathrm{Ct}}$ value (Schmittgen et al.,2008).

208

209

210

211 One hour after imposing, the content of carbohydrate, $\mathrm{H}_{2} \mathrm{O}_{2}$ and total protein in both Salt-

212 sensitive and Salt-tolerant cultivars were determined. The results showed that no significant

213 change was observed in the content of carbohydrate among both cultivars (Fig.1A). Moreover,

214 there was no significant difference in the content of $\mathrm{H}_{2} \mathrm{O}_{2}$ between in Salt-tolerant tomato 
215 compared with the Salt-sensitive tomato (Fig.1B). One hour after imposing, the content of $\mathrm{H}_{2} \mathrm{O}_{2}$

216 in Salt-sensitive tomato was still significantly higher than that in Salt-tolerant cultivars (Fig.1B),

217 which may cause damage to these cultivars. In parallel, there was no significant difference in

218 content of total protein among both Salt-sensitive and Salt-tolerant cultivars. However, $1 \mathrm{~h}$ after

219 imposing, the content of total protein in Salt-tolerant cultivars was significantly increased to a

220 higher level than Salt-sensitive cultivars (Fig.1C).

\section{Global proteome analysis of Salt tolerant cultivars (ST) and Salt sensitive (SS) cultivars}

222 To further elucidate the mechanism of resistance to salt stress, mass spectroscopy based

223 proteomics was performed. Both Salt tolerant cultivar (ST) and Salt sensitive (SS) cultivar

224 treated after 0h (ST and SS), $1 \mathrm{~h}$ (ST 1h and SS 1h) and 12h (ST 12h and SS 12h) were harvested

225 for proteomic analysis, and each treatment had three replicates. Furthermore, 7663 function

226 proteins were identified among all samples and analyzed by variation analysis (Supplementary

227 Table 2).

228 To investigate the influences of salt stress on cultivars (SS and ST), two algorithms were further

229 performed, including principle components analysis (PCA) and unsupervised cluster analysis

230 (Fig.2A, 2B). First, PCA analysis showed an observable separation between all six treatments

231 demonstrating that salt stress induced significant changes in proteomic profiles of both cultivars

232 (SS and ST) and the responses to salt stress of ST were different to SS (Fig.2B). Meanwhile,

233 unsupervised cluster analysis showed a stability of replicates from each sample groups,

234 validating our treatments (Fig.2A). It further reach a consensus with PCA analysis that all

235 replicates with same treatments clustered together and observably separated with each other. We

236 further performed a two-factor (time point and strains) PCA analysis (Fig.2C,2D) on these

237 proteomic data sets to detected the response to salt stress at different time point. Focus on the

238 time points, we found that the most active responses were presented at $1 \mathrm{~h}$ after imposing

239 (Fig.2C). Interestingly, the significant changes at 0h indicated that the basal level of proteomic

240 profile between SS and ST were different with each other (Fig.2D). Therefore, we proposed that

241 the basal level of some salt resistance-related protein of ST were different with SS, resulting in a 
242 strong tolerance of ST to salt stress. Meanwhile, the ST can further make some responses under

243 salt stress at $1 \mathrm{~h}$, which is superior than SS in response to salt stress.

244 Proteins located at chloroplast exhibited significant difference Salt tolerant cultivar (ST) 245 and Salt sensitive (SS) cultivar

246 To investigate the differences in proteome between SS and ST before salt stress, we compared

247 the proteomic profiles of ST and SS before treatment. Among 7663 identified proteins, only 248 protein in which the $\log _{1.5}$ (fold change) $>1$ or $<-1$ and the $\mathrm{P}$ value $<0.05$ were identified as 249 differentially expressed proteins (DEPs; Fig.3A). Finally, 278 proteins matched the condition 250 were identified as DEPs (Fig.3A). Among 278 DEPs, there are 131 up-regulated proteins and 251147 down-regulated proteins in ST compared to SS (Fig.3A). Stress-regulated genes, such as the 252 lipoxygenase (A0A3Q7ENA4), superoxide dismutase (Q7XAV2) and gamma aminobutyrate 253 transaminase 2 (A0A3Q7J2V8) were up-regulated in ST compared to their expression in SS, 254 suggesting that ST have more advantages in resistance to salt stress than SS. Meanwhile, we 255 found that the activity of two stress-related enzyme, SOD and PPO, were more active in ST than 256 that in SS, whereas the enzyme of other two stress-related enzyme, POD and PAO, did not 257 exhibited differences in activity in both SS and ST (Fig.3B, 3C). We further analyze the location 258 of these DEPs, the results indicated that most of differentially expressed proteins were located at 259 chloroplast, cytoplasm, nucleus, extracellular, plasma membrane and mitochondria, 260 demonstrating the proteins functioned at membrane and chloroplast contributed the differences 261 of resistance to salt stress between SS and ST (Fig.3E). It has been reported that various proteins 262 at chloroplast and membrane play key role in resistance to salt stress, including Superoxide 263 dismutase (Q7XAV2) and gamma aminobutyrate transaminase 2 (A0A3Q7J2V8; Arora et al., 264 2002; Blokhina et al., 2003). Therefore, we concluded that the basal resistance of ST to salt 265 stress was higher than SS.

266 Gene enrichment analysis for DEGs from ST and SS

267 To study the differences of biological functions related to the diverse responses between SS and 
268 ST, we performed a Gene ontology enrichment analysis on all 278 DEPs using TopGO (Fig4;

269 Supplementary Table 3). A total of 34 GO terms relevant to molecular progresses were 270 significantly enriched based on these 278 DEPs, including heme binding, tetrapyrrole binding,

271 peroxidase activity, oxidoreductase activity, peroxide acceptor, chitinase activity, antioxidant 272 activity, intramolecular lyase activity and catalytic activity (Fig. 4A). Various terms were 273 involved in the response to abiotic stress, such as peroxidase activity, oxidoreductase activity and 274 chitinase activity. In addition, among identified cellular component-related terms, 20 terms were 275 significantly enriched such as extracellular region, MCM complex, protein-DNA complex, 276 replisome, nuclear replication fork and nuclear replisome (Fig. 4A). Under salt stress, a bunch of 277 reactive oxygen species (ROS) could be produce in plants which cause damage to DNA and 278 membrane (GONG et al.,2013; Singh et al., 2016; Scott et al.,1987). Therefore, proteins having 279 DNA-, ROS- and membrane-related function could protect the DNA from damage, ensuring 280 normal biological processes. Finally, compared to SS, the results of GO enrichment analysis 281 further showed that 125 terms relevant to biological process category were significantly enriched 282 ( $\mathrm{P}<0.05$; Fig.4A). Remarkably, many stress-related biological processes were significantly 283 identified, including response to stress, reactive oxygen species metabolic process, hydrogen 284 peroxide metabolic process, response to stimulus, chitin metabolic process, cell wall 285 macromolecule catabolic process, response to oxidative stress, response to external stimulus and 286 cellular carbohydrate metabolic process (Fig. 4A), indicating that the resistance response to salt 287 stress were different between SS and ST. Moreover, the results of KOG analysis on these DEPs 288 showed that the expression of proteins which involved in signal transduction mechanisms, 289 energy production and conversion, carbohydrate transport and metabolism, amino acid transport 290 and metabolism, lipid transport and metabolism, secondary metabolites biosynthesis, transport 291 and catabolism were significantly altered in ST compared to SS (Fig. 4B). It has been proved that 292 amino acids such as proline and lipids such as glyceride play important functions in salt 293 resistance of plants (Ashraf et al., 2007; Butt et al., 2016; Huang et al., 2014). In parallel, signal 294 transduction also influence the plant salt resistance, such as hormone JA and ABA (Msanne et al., 
295 2011; MLLER et al., 2015; Zhu et al., 2010; LI et al., 2015; Huang et al., 2012; Guo et al., 2015).

296 Various studies have proved the important function of JA and ABA in strong resistance

297 responses of plants to salt stress (Ding et al., 2016; Me et al., 2016; ISLAM et al., 2017; Jiang et

298 al., 2016; LIU et al., 2015; GURMANI et al., 2013). In the pathways analysis, various

299 biosynthesis relevant to JA and ABA were significantly enriched, including $\alpha$-linoleic acid

300 biosynthesis and steroid biosynthesis (Fig. 4A). Based on these results above, we proposed that

301 ST have more advantages in resisting salt stress compared to SS.

302 Proteins involved in various salt resistance-related pathways were altered in ST

303 Similarly, pathway enrichment analysis were also performed on all DEPs against KEGG

304 database. The results showed that there were 84 biosynthesis pathways were enriched (Fig.5A).

305 Among 84 pathways, 14 pathways were significantly enriched (Fig.5A; $\mathrm{P}<0.05$;

306 Supplementary Table 4), including Phenylpropanoid biosynthesis, Metabolic pathways,

307 Flavonoid biosynthesis, biosynthesis of secondary metabolites, DNA replication, Nitrogen

308 metabolism, Valine, leucine and isoleucine degradation and Zeatin biosynthesis (Fig.5A). It has

309 been reported that secondary metabolites played important roles in response to salt stress

310 (Hazman et al., 2015). The increase in content of betaine and sorbital could enhance the

311 resistance of various varieties to salt stress (Orthen et al., 1994; Kanayama et al., 2007; Gao et al.,

312 2000; Fu et al., 2011). Remarkably, all these metabolites-related pathways were significantly

313 reprogrammed in ST (Fig.5A), which may contribute to the advantages of ST in response to salt

314 stress. Based on these three function analyses, 112 DEPs which functioned in these terms were

315 identified (Fig.5B). In the expression pattern of these DEPs, two modules were constructed in the

316 heatmap (Fig.5B). Among these 112 DEPs, 76 were up-regulated in ST and clustered at the top

317 module which was labeled by pink square, whereas 36 proteins were down-regulated (Fig.5B).

318 Among highly up-regulated proteins, Superoxide dismutase (Q7XAV2), Lipoxygenase

319 (A0A3Q7ENA4) and various proteins relevant to chloroplast were involved in plant resistance

320 response to salt stress (Smirnoff, 2005; Andronis \& Roubelakis- Angelakis, 2010; Schreiner \& 
321 Zozor, 1996). All these 76 up-regulated DEPs were further recognized as key proteins which

322 were important for the account for the mechanism of stronger salt resistance of ST than SS.

323 Responses of ST and SS were different under salt stress

324 To further clarify the advantage of ST in response to salt stress, we compared the proteomic 325 profiles of ST and SS after 1h imposing, which was observably separated with each other in PCA 326 plots. In ST1.vs.SS1 pairwise comparison, 169 proteins were identified as DEPs (Fig.6A). 327 Among 169 DEPs, 49 proteins were up-regulated in ST, whereas 120 proteins were up-regulated 328 in SS (Fig.6A). The expression level of various proteins functioned in response to salt stress 329 were higher than SS, including Photosystem II reaction center protein, non-specific lipid-transfer 330 protein 2-like, $\mathrm{ABC}$ transporter $\mathrm{F}$ family member 5, anion: sodium symporter and gamma 331 aminobutyrate transaminase 2 . It has been proved that high expression level of lipid-transfer 332 protein could enhance the resistance of plants to salt stress, and such protein could also be 333 induced to up-regulated under salt stress (Li, 2004; Torres-Schumann et al., 1992). Interestingly, 334 all the DEPs were still mainly located at chloroplast, nucleus, cytoplasm, extracellular and 335 plasma membrane (Fig.6E). Remarkably, after 1h imposing, the enzyme activity of POD, PAO 336 and PPO were further activated in ST, whereas the activity of these three stress-related enzyme 337 were decreased in SS, which account for the advantage of ST in tolerance of salt challenge 338 (Table 1). However, the activity of SOD did not display significant changes in SS following salt 339 stress challenges (Table 1). Overall, all the 49 up-regulated DEPs were recognized as focus for 340 further analysis below to clarify the advantage of ST.

\section{The advantage of ST in response to salt stress}

342 To elucidate the advantage of ST in response to salt stress after $1 \mathrm{~h}$ imposing, we further 343 performed function analysis. Compared to SS, all the up-regulated DEPs in ST after 1h imposing 344 were analyzed by Gene Ontology enrichment analysis (Fig. 7A, 7B, 7C). The results showed that 345 many significantly enriched molecular function-related terms were involved in salt stress, which 
346 were helpful for plants to tolerate salt stress, including electron transporter, transferring electrons

347 within the cyclic electron transport, pathway of photosynthesis activity, structural constituent of

348 cytoskeleton, 4-aminobutyrate transaminase activity, ATP:ADP antiporter activity, heat shock

349 protein binding (Fig. 7B). Enzymes with ATPase activity perform important roles in plant

350 resistance to salt stress (Rangani et al., 2016). Meanwhile, aminobutyric acid is also an important

351 chemical component in plants response to salt stress, and 4-Aminobutanoate (GABA) can

352 alleviate the influences of salt stress on a variety of plants, including tomato, corn and wheat (Li

353 et al., 2016; Luo et al., 2012; Shi et al., 2010). For cellular component categorizes, gene

354 functioned in chloroplast-related terms were highly up-regulated in ST under salt stress, 355 including chloroplast, chloroplast part and chloroplast thylakoid (Fig. 7A). Under salt stress,

356 chloroplast could help plant scavenge the excessive accumulation of ROS and maintain the

357 normal growth of plants (Li et al.,2016; Lu et al.,2006). Furthermore, the significantly enriched

358 biological progresses relevant to salt stress were response to wounding, cellular carbohydrate

359 metabolic process, phloem development, cell wall biogenesis, response to stimulus and response

360 to hydrogen peroxide (Fig. 7C). The strengthened cell wall and carbohydrate metabolites are

361 important for plants as being challenged by salt stress (Pang et al., 2016; Dinneny et al., 2015;

362 Liu et al., 2015; Khan et al., 2016). Further pathway analysis on 169 DEPs showed an

363 overrepresentation of pathways relevant to resistance to salt stress, such as Alanine, aspartate and

364 glutamate metabolism, alpha-Linolenic acid metabolism, Linoleic acid metabolism, Peroxisome,

365 Steroid biosynthesis, Valine, leucine and isoleucine degradation (Fig.7D). Amino acid proline

366 could contribute the tolerance of plants to salt stress by functioning as a protective agent and

367 nitrogen source for cell structure and enzymes (Mansour et al., 1998; Butt et al., 2016).

368 Meanwhile, steroid biosynthesis could increase the content of ABA, which was widely identified

369 in plant response to salt stress and played important function in these processes (Albacete et al.,

370 2008, 2010). Remarkably, 21 proteins were identified from these important pathways and Go

371 function terms, including Lipoxygenase (Q84P54), gamma aminobutyrate transaminase 2

372 (A0A3Q7H8R6), Peroxidase (A0A3Q7EK65) and Glutamine synthetase (A0A3Q7J2V8) 
373 (Fig.7E). Protein Peroxidase have been widely recognized as important role in salt resistance.

374 Therefore, we proposed that the advantage of ST in response to salt stress was mainly activation

375 of salt resistance-related metabolisms and alteration of cell structure.

\section{Three key function proteins were important for the salt tolerance of ST}

377 Overall, we found that three up-regulated proteins were shared in both ST.vs.SS and ST1.vs.SS1

378 pairwise comparisons, including A0A0C6G3Q8, A0A3Q7J2V8 and A0A3Q7IRF5 (Fig.8A;

379 Table2). Both A0A3Q7J2V8 and A0A3Q7IRF5 perform function at chloroplast, whereas

380 A0A0C6G3Q8 is located at plasma membrane (Table 2). Remarkably, all these 3 proteins up-

381 regulated 1.8 2.0-fold in ST.vs.SS pairwise comparison (Fig.8C, 8D, 8E). And their expression

382 in SS following $1 \mathrm{~h}$ imposing was still almost 2.0 -fold lower than ST after 1h imposing

383 (Fig.8C,8D, 8E). However, all of them could be induced to up-regulated in SS after 1h imposing

384 (Fig.8C, 8D,8 E). By function analysis of each proteins, we found that A0A0C6G3Q8 has Sterol

385 side chain reductase function, and A0A3Q7J2V8 is gamma aminobutyrate transaminase, whereas

386 A0A3Q7IRF5 involved in Starch synthase (Table2). Remarkably, up-regulation of

387 A0A0C6G3Q8 could promote the synthesis of Brassinolide in vivo, and the increase in content

388 of brassinolide may also contribute the plant resistance to salt stress and freezing injury, which

389 has been widely used in agriculture (Uday et al., 2010; Xia et al., 2009; Hua et al., 2011).

390 Additionally, up-regulation of A0A3Q7J2V8 encoding gamma aminobutyrate transaminase

391 promotes synthesis of GABA which has been widely used in filed to enhance plant resistance to

392 various abiotic stress (Li et al., 2016; Shi et al., 2010). Importantly, in the co-expression network

393 we further observed the hub role of these 3 proteins that all of them were located at the core of

394 the network and closely correlated with other up-regulated proteins which involved in resistance

395 to salt stress (Fig.8B). qRT-PCR analysis was further used for validation of these three key

396 proteins of ST in response to salt stress. The results showed there was no significant differences

397 in basal expression level of these three genes in both SS and ST cultivars. In contrast, dramatic

398 up-regulation of these genes was induced by 1 hour imposing in ST cultivars, whereas their 
399 expression levels were not altered in SS after 1 hour imposing, leading a strong tolerance of ST 400 to salt stress (Fig 9). Therefore, we highlight the importance of A0A0C6G3Q8, A0A3Q7J2V8

401 and A0A3Q7IRF5 in the mechanism of resistance of ST to salt stress.

\section{DISCUSSION}

403 Salt stress seriously influences growth and development of tomato, which seriously restricts the 404 yield of tomato. With the development of researches, it has been showed that plants can resist 405 salt stress by various physiological changes. Among our both ST and SS cultivars, the activity of 406 SOD, PPO and POD was higher in ST cultivars, leading a strong tolerance to salt stress. 407 Subsequently, in this study, the changes in proteomic profiles of salt-tolerant and salt-sensitive 408 tomato varieties were compared. We found that salt-tolerant cultivars and salt-sensitive cultivars 409 had significantly different responses to salt stress.

410 Salt-tolerant cultivars could enhance the tolerance of plants to salt stress by changing the 411 expression of membrane structure-related proteins. Meanwhile, a variety of metabolic pathways 412 were altered between salt-tolerant and salt-sensitive cultivars. Compared with the Salt-sensitive 413 cultivars, there is a higher active pattern of metabolisms relevant to Nitrate and amino acids. And 414 under the challenge of salt stress, the salt-tolerant cultivars can further activated the 415 Brassinolides and GABA biosynthesis, which contribute to the resistance to salt stress. 416 Importantly, the higher expression of chloroplast-related protein in salt-tolerant cultivars 417 indicated that salt-tolerant cultivars had higher photosynthetic activity, which could 418 promotenormal growth of plants under salt stress. Additionally, Salt-tolerant plants have 419 advantages in scavenging reactive oxygen species produced by salt stress. A bunch of 420 antioxidant-related proteins were activated to up-regulated in Salt-tolerant cultivars, such as 421 peroxidase and Catalase isozyme. In-depth analysis, we identified three key proteins, which

422 highly expressed in Salt-tolerant cultivars and play key role in its strong resistance to salt stress, 423 including Sterol side chain reductase, gamma aminobutyrate transaminase and Starch synthase.

424 N-related metabolisms play important function in salt-tolerance of plants. A remarkable property 425 of salt-tolerant is that the proteins involved in N metabolism were highly up-regulated in ST. It 
426 has been documented that high value of $\mathrm{pH}$ caused by salt stress will significantly influence the $427 \mathrm{~N}$ metabolism and absorption of plants (Groppa et al., 2008; Shan et al., 2016). The absorption of $428 \mathrm{NO}_{3}^{-}$by plants is mainly regulated by $\mathrm{H}^{+} / \mathrm{NO}_{3}^{-}$co-transporters, which requires high concentration 429 of protons around the channel as the transport energy (Dluzniewska et al., 2007). Under the salt 430 stress, the high $\mathrm{pH}$ environment around the roots leads to the shortage of protons around the 431 channels, which greatly restricts the assimilation or absorption of $\mathrm{NO}_{3}^{-}$by the roots, and inhibits 432 the transport of $\mathrm{NO}_{3}^{-}$, resulting in the decrease of the content of $\mathrm{NO}_{3}^{-}$in the roots, thus 433 interfering the whole nitrogen metabolism pathway (Abouelsaad et al., 2016; Murtaza et al., 434 2013; Shao et al., 2015). Our proteomic profiles of Salt-tolerant and Salt-sensitive cultivars 435 revealed that various proteins involved in N metabolism were highly up-regulated in Salt-tolerant 436 cultivars, in contrast these proteins were down-regulated in Salt-sensitive cultivars such as AMT 437 and NRT. Some studies have shown that plant roots transport $\mathrm{NH}_{4}{ }^{+}$through AMT protein family, 438 and transport $\mathrm{NO}_{3}{ }^{-}$by NRT protein family. Under salt stress, the expression levels of OsNRT 439 and most members of OsAMT family in rice roots are increased, for the increase in absorption of $440 \mathrm{NO}_{3}{ }^{-}$and $\mathrm{NH}_{4}{ }^{+}$to make up for the deficiency of content in roots. These results suggested that the $441 \mathrm{~N}$ metabolism were not impaired in Salt-tolerant cultivars, which maintains the normal 442 physiological requirements of plants and enhance the tolerance of plants to salt stress. Therefore, 443 one of the advantages of Salt-tolerant cultivars was relative high levels of $\mathrm{N}$ metabolism. 444 Similarly, the salt-tolerant rice were also exhibited high level of N metabolism, which contribute 445 to its tolerance to salt stress (Lutts et al., 1999). Meanwhile, it also has been proved that the 446 amino acids-, carbohydrate- and flavonoids-related biosynthesis also performed important 447 function in the adaption of plants to salt stress.

448 Subsequently, our proteomic data showed a high expression level of genes relevant to GABA 449 and brassinolides biosynthesis. BR can improve plant resistance to biological and abiotic stress 450 (Uday et al., 2010; Hua et al., 2011). The regulation of BR on plant stress resistance was closely 451 related to the accumulation of $\mathrm{H}_{2} \mathrm{O}_{2}$ and the enhancement of the activity of antioxidant enzymes 452 (SOD, CAT, APX; Xia et al., 2009; Zhang et al., 2010). Exogenous BR can significantly 
453 improve the growth and development of seedling plants under salt stress, reduce salt damage 454 index, and significantly increase the content of proline and soluble sugar in leaf cells and the 455 activity of antioxidant enzymes such as SOD, POD and CAT (Shang Qingmao et al., 2006), thus 456 improving the salt resistance of cucumber seedlings. Meanwhile, exogenous BR can improve the 457 salt tolerance of Arabidopsis thaliana (Hua et al., 2011). Under salt stress, exogenous EBR could 458 promote the activities of CAT and SOD enzymes in tomatoes, reduce the content of MDA in 459 cells, and improve the salt tolerance of tomatoes (Ahmmed et al., 2013). Gamma-aminobutyric 460 acid also plays an important role in plant salt tolerance, GABA can regulate cytoplasmic $\mathrm{pH}$, 461 resist oxidative stress, interact with microorganisms, and defend insects as osmotic regulator and 462 signal molecule (Gilliham et al., 2016; Bouché et al., 2004). Appropriate concentration of GABA 463 can effectively promote seed germination and seedling growth under salt stress (Ziogas et al., 464 2017). GABA can reduce oxidative damage by improving the antioxidant capacity of plants (Li 465 et al., 2016). At the same time, it can improve the content of osmotic regulation substances, 466 maintain osmotic balance ( $\mathrm{Li}$ et al., 2010), inhibit photosynthetic pigment degradation, and 467 maintain photosystem II (PSII) activity. In addition, GABA can further regulate plant hormone 468 content (Shi et al., 2010) to improve plant salt tolerance. Therefore, high basal level of BRs and 469 GABA biosynthesis will be a new direction for breeding salt-tolerant cultivars.

470 Chloroplast were also important for the tolerance of plants to salt stress. Under abiotic stress, the 471 most vulnerable part is PSII (Wei et al., 2012). The photosynthetic rate decreased due to salt 472 stress, and salt stress would also reduce leaf turgor pressure and cause partial stomatal closure to 473 further inhibit photosynthesis (Chaves et al., 2009). Our data showed a high expression pattern of 474 proteins which involved in chloroplast membrane, demonstrating that the Salt-tolerant cultivars 475 have advantages in response to salt stress challenge. The salt-tolerant cultivars showed an 476 overrepresentation of chloroplast-related terms, which indicated that a bunch of proteins 477 functioned in chloroplast were purposefully highly expressed. The reason is that plants need to 478 maintain the photosynthesis of plants at normal level, which providing adequate nutrient for 479 plants to enhance tolerance to salt stress. Additionally, various hormone-related biosynthesis 
480 were also closely correlated with the chloroplast, such as ABA, JA and GA3. Meanwhile, plant 481 hormones play important roles in the signal transduction of plants in response to salt-stress, 482 including ABA and BRs (Yang et al., 2013; Dodd et al., 2009). Hormone synthesis is induced by 483 various abiotic stress, and their content varies with the degree of salt-stress. The changes in 484 content of hormone will initiate or regulate some physiological and biochemical processes 485 relevant to the response to abiotic stress. Researches have indicated that exogenous application 486 of ABA will enhance the tolerance of plants to salt stress (Dodd et al., 2009). We noted that both 487 biosynthesis of ABA and BRs were activated in Salt-tolerant cultivars, whereas these 488 biosynthesis exhibited an low level of activity in Salt-sensitive cultivars.

489 The ROS scavenging system is imperative for plant growth and development (Katalin et al., 490 2011; Hajiboland et al., 2010). Under salt stress challenge, Plants always improve their salt491 tolerance by regulating the dynamic balance of reactive oxygen species (ROS), which is caused 492 by salt stress and results in damage to plants. In our research, we found that proteins involved in 493 ROS scavenging system was highly expressed in salt-tolerant tomato, suggesting that the salt494 tolerant cultivars have more advantages in scavenging of ROS, whose accumulation was caused 495 by salt stress, than salt-sensitive cultivars. It has been proved that excessive accumulation of 496 reactive oxygen species will damage the integrity of the plant cell membrane system and 497 influence the plants development and growth (Smirnoff, 1993; Asada, 1999; Shigeoka et al., 498 2002). Remarkably, excessive ROS accumulation will also cause damage to the ultrastructure of 499 chloroplast, which influences the photosynthetic system (Katalin et al., 2011). In parallel, it also 500 could inhibit the activity of photosynthesis-related enzyme, leading a decrease in efficient of 501 photosynthetic system (Katalin et al., 2011). In contrast, we have provided full evidences that the 502 chloroplast and photosynthesis of salt-tolerant cultivars were more active than that in salt503 sensitive cultivars. Active photosynthesis also could contribute to the scavenging of ROS by 504 activation of SOD (Arora et al., 2002; Andronis et al., 2010). Similar to our results, it has been 505 proved that there is less accumulation of ROS in the salt-tolerant cultivars, with high 506 photosynthesis in vivo. Therefore, we further recognized the highly active ROS scavenging 
507 system as an important advantage of Salt-tolerant tomato as being challenges by salt stress.

508 Soil salinization has greatly restricted the production and development of agriculture. Breeding

509 salt-tolerant cultivars is still believed to be the most cost-effective strategy to deal with the

510 influence of salt stress on crops. However, the limited resources of salt-tolerant genes restrict

511 the breeding of new tomato varieties. In our study, by comparative proteomic analysis, we found

512 that the advantages of Salt-tolerant tomato in response to salt stress were mainly high level of N-

513 and ABA-related biosynthesis, effective photosynthesis system and active ROS scavenging

514 system, leading a strong tolerance of tomato to salt stress. Additionally, high level of GABA and

515 Brassinolides biosynthesis also contribute the strong tolerance of tomato, and exogenous 516 application of both metabolites can be applied in yield. In parallel, three genes playing important

517 role in salt-resistance of Salt-tolerance tomato were further identified, including A0A3Q7J2V8,

518 A0A3Q7IRF5 and A0A0C6G3Q8, which can be used in future. Additionally, GABA and

519 Brassinolides were also involved in the plant resistance to salt stress. In summary, our results 520 provide mechanistic insights into the advantages of salt-tolerant cultivars vs. salt-sensitive 521 cultivars via comparative proteomic analysis.

\section{CONCLUSIONS}

523 Our results suggested that salt-tolerant cultivars have more advantages in resistance to salt stress 524 than that of salt-sensitive cultivars. The abundance and activity of antioxidant-related proteins, 525 such as SOD, PPO and POD, were higher in the salt-tolerant cultivars, leading effective 526 advantages in dealing with reactive oxygen species caused by salt stress. Meanwhile, higher 527 expression level of proteins relevant to Nitrate and amino acids metabolisms and photosynthetic 528 activity could guarantee a sufficient nutrient for the growth of salt-tolerant under salt stress.

529 Three key proteins functioned in Brassinolides and GABA biosynthesis, including Sterol side 530 chain reductase, gamma aminobutyrate transaminase and Starch synthase performed important 531 roles in regulation and activation of salt-resistance of salt-tolerant cultivars.

\section{ACKNOWLEDGEMENTS}

533 We would like to thank PTM-Biolabs Co., Ltd. (Hangzhou, China) for the quantitative proteomic 
534 analysis assistance.

535 This work was supported by the National Natural Sciences Foundation of China (31860554).

536 ADDITIONAL INFORMATION AND DECLARATIONS

537 Funding

538 This work was funded by the National Natural Science Foundation of China, Grant No.31860554,

539 No.31360482

\section{Competing Interests}

541 The authors declare that they have no competing interests

\section{Author Contributions}

543 - Qiang Wang conceived and designed the experiments, analyzed the data, contributed

544 reagents/materials/analysis tools, prepared figures and/or tables, approved the final draft.

545 - Baike Wang performed the experiments, contributed reagents/materials/analysis

546 tools,prepared figures and/or tables, approved the final draft.

547 - Huifang Liu, performed the experiments, contributed reagents/ analysis tools,prepared

548 figures and/or tables, approved the final draft.

549 - Hongwei Han analyzed the data, authored or reviewed drafts of the paper, approved the final 550 draft.

551 - Hongmei Zhuang analyzed the data, authored or reviewed drafts of the paper, approved the 552 final draft.

553 - Juan Wang analyzed the data, authored or reviewed drafts of the paper, approved the final 554 draft.

555 - Tao Yang analyzed the data, authored or reviewed drafts of the paper, approved the final 556 draft

557 - Hao Wang conceived and designed the experiments, performed the experiments, analyzed 558 the data, prepared figures and/or tables, authored or reviewed drafts of the paper, and 559 approved the final draft.

560 - Yong Qin conceived and designed the experiments, performed the experiments, analyzed the 
561 data, prepared figures and/or tables, authored or reviewed drafts of the paper, and approved

562 the final draft.

563 Data Availability

564 The datasets generated for this study can be found in the ProteomeXchange

565 (https://www.iprox.org) with the accession number PXD029130.

566 REFERENCES

567 Abouelsaad I, Weihrauch D, Renault S. 2016.Effects of salt stress on the expression of key 568 genes related to nitrogen assimilation and transport in the roots of the cultivated tomato and 569 its wild salt-tolerant relative. Sci Hortic-Amsterda 211: 70-78

570

571 DOI:10.1016/j.scienta.2016.08.005.

Ahammed G J,Ruan Y P, Zhou J,Xia X J, Shi K,Zhou Y H,Yu J Q. 2013. Brassinosteroid 572

573

574

575

576

577

578

579

580

581

582

583

584

585

586

587

588

589

590

591

592 alleviates polychlorinated biphenyls-induced oxidative stress by enhancing antioxidant enzymes activity in tomato. 90(11):2645-2653 DOI: 10.1016/j.chemosphere.2012.11.041.

Albacete A, Ghanem M E, Dodd I C , Pérez-Alfocea F.2010. Principal component analysis of hormone profiling data suggests an important role for cytokinins in regulating leaf growth and senescence of salinized tomato. Plant Signaling \& Behavior 5(1) :45-48 DOI: $10.4161 / p s b .5 \cdot 1.10120$.

Albacete A, Ghanem ME, Martínez-Andújar C, Acosta M, Sánchez-Bravo J,Martínez V, Lutts S, Dodd IC, Pérez-Alfocea F. 2008. Hormonal changes in relation to biomass partitioning and shoot growth impairment in salinized tomato (Solanum lycopersicum L.) plants. J Exp Bot 59(15):4119-4131 DOI: 10.1093/jxb/ern251.

Allakhverdiev Suleyman I,Nishiyama Yoshitaka, Miyairi Sachio, Yamamoto Hiroshi,Inagaki Noritoshi, Kanesaki Yu, Murata Norio. 2002.Salt Stress Inhibits the Repair of Photodamaged Photosystem II by Suppressing the Transcription and Translation of psbAGenes in Synechocystis. Plant Physiology 130(3) 1443-1453 DOI: 10.1104/PP.011114.

Andronis E A, Roubelakis-Angelakis K A. 2010. Short-term salinity stress in tobacco plants leads to the onset of animal-like PCD hallmarks in planta in contrast to long-term stress. Planta 231 : 437-448 DOI: 10.2307/23391038.

Arora A, Sairam R K, Srivastava G C. 2002. Oxidative stress and antioxidative system in plants. Current Science, 82: 1227-1238.

Asada K. 1999. The water-water cycle in chloroplasts: Scavenging of active oxygen and 593 dissipation of excess photons. Annual Review of Plant Physiology and Plant Molecular Biology50: 601-639 DOI: 10.1146/annurev.arplant.50.1.601. 
595

596

597

598

599

600

601

602

603

604

605

606

607

608

609

610

611

612

613

614

615

616

617

618

619

620

621

622

623

624

625

626

627

628

629

630

Ashraf M, Foolad M R. 2007. Roles of glycine betaine and proline in improving plant abiotic stress resistance.Environmental and Experimental Botany 59(2):206-216 DOI: 10.1016/j.envexpbot.2005.12.006.

Blokhina O, Virolainen E, Fagerstedt K V. 2003. Antioxidants, oxidative damage and oxygen deprivation stress: A review. Annals of Botany 91: 179-194 DOI: 10.1093/aob/mcf118.

Bouché N, Fromm H. 2004.GABA in plants: just a metabolite?. Trends Plant Sci 9(3): 110115 DOI: 10.1016/j.tplants.2004.01.006.

Bruns S, Hecht-Buchholz C.1990. Licht-und elektronenmikroskopische Untersuchungen an Blättern mehrerer Kartoffelsorten nach Salzgaben zu verschiedenen Entwicklungsstadien. Potato Research, 33(1), 33-41. DOI:10.1007/bf02358128.

Butt M, Ayyub C M.Amjad M, Ahmad R. 2016. Proline application enhances growth of chilli by improving physiological and biochemical attributes under salt stress.Pakistan Journal of Agricultural Sciences 53 (1) :43-49.

Chartzoulakis K S, Loupassaki M H. 1997. Effects of $\mathrm{NaCl}$ salinity on germination, growth, gas exchange and yield of greenhouse eggplant. Agricultural Water Management, 32(3), 215-225. DOI:10.1016/s0378-3774(96)01276-0.

Chaves M M, Flexas J, Pinheiro C. 2009. Photosynthesis under drought and salt stress: regulation mechanisms from whole plant to cell. Ann Bot 103(4): 551-560 DOI: 10.1093/aob/men125.

CHEN W C.2011.Physilogical mechanisms of alkali tolerance in three economic plants.Northeast Normal University.

Chunwu Yang Jianna ChongChangyou LiChangmin KimDecheng ShiDeli Wang.2007. Osmotic adjustment and ion balance traits of an alkali resistant halophyte Kochia sieversiana during adaptation to salt and alkali conditions.Plant and Soil 294:263-276 DOI: $10.1007 / \mathrm{s} 11104-007-9251-3$.

Colcombet J, Hirt H. 2008. Arabidopsis MAPKs: a complex signalling network involved in multiple biological processes. Biochemical Journal, 413(2), 217-226. DOI: 10.1042/bj20080625.

Cui D, Wu D, Liu J, Li D, Xu C, Li S, et al.2015. Proteomic Analysis of Seedling Roots of Two Maize Inbred Lines That Differ Significantly in the Salt Stress Response. PLoS ONE 10(2): e0116697.DOI:10.1371/journal.pone.0116697.

Ding H, Lai J B, Wu Q, Zhang S C, Chen L, Dai Y S, Wang C F, Du J J, Xiao S, Yang C W. 2016.Jasmonate complements the function of Arabidopsis lipoxygenase 3 in salinity stress response.Plant Science 244:1-7 DOI: 10.1016/j.plantsci.2015.11.009.

Dinneny J R.2015.Traversing organizational scales in plant salt-stress responses. Current 
631 Opinion in Plant Biology 23:70-75 DOI: 10.1016/j.pbi.2014.10.009.

632 Dluzniewska P, Gessler A, Dietrich H, Rennenberg • 2007.Nitrogen uptake and

633

634

635

636

637

638

639

640

641

642

643

644

645

646

647

648

649

650

651

652

653

654

655

656

657

658

659

660

661

662

663

664

665

666 metabolism in Populus x Canescens as affected by salinity. New Phytol 173(2): 279-293 DOI: $10.2307 / 4131248$.

Dodd I C, Theobald J C, Richer S K, Davies W J. 2009. Partial phenotypic reversion of ABA-deficient flacca tomato (Solanum lycopersicum) scions by a wild-type rootstock: normalizing shoot ethylene relations promotes leaf area but does not diminish whole plant transpiration rate. Journal of Experimental Botany 60(14): 4029-4039 DOI: 10.1093/jxb/erp236.

Fu Xing-zheng, Khan E U, Hu Shuang-shuang, Fan Qi-jun, Liu Ji-hong. 2011. Overexpression of the betaine aldehyde dehydrogenase gene from Atriplex hortensis enhances salt tolerance in the transgenic trifoliate orange (Poncirus trifoliata L. Raf.) . Environmental and Experimental Botany 74: 106-113 DOI: 10.1016/j.envexpbot.2011.05.006.

Faizan Mohammad; Bhat Javaid Akhter; Chen Chen; Alyemeni Mohammed Nasser; Wijaya Leonard; Ahmad Parvaiz; Yu Fangyuan.2021. Zinc oxide nanoparticles (ZnO-NPs) induce salt tolerance by improving the antioxidant system and photosynthetic machinery in tomato. Plant Physiology and Biochemistry 161:122-130 DOI: 10.1016/J.PLAPHY.2021.02.002.

Gao M, Sakamoto A, Miura K, Murata N, Sugiura A, Tao R. 2000. Transformation of Japanese persimmon (Diospyros kaki Thunb.) with a bacterial gene for choline oxidase. Molecular Breeding 6: 501-510 DOI: 10.1023/a:1026513831290.

Gilliham M, Tyerman S D. 2016.Linking metabolism to membrane signaling: the GABAmalate connection. Trends Plant Sci 21(4): 295-301 DOI: 10.1016/j.tplants.2015.11.011.

Gong B, WenD, VandenLangenberg K, Wei M, Yang F, Shi Q,Wang X. 2013. Comparative effects of $\mathrm{Na} \mathrm{Cl}$ and $\mathrm{Na} \mathrm{HCO}_{3}$ stress on photosynthetic parameters, nutrient metabolism, and the antioxidant system in tomato leaves.Scientia Horticulturae 157:1-12DOI: 10.1016/j.scienta.2013.03.032.

Gong, B., Zhang, C., Li, X., Wen, D., Wang, S., Shi, Q., \& Wang, X. 2014. Identification of $\mathrm{NaCl}$ and $\mathrm{NaHCO}_{3}$ stress responsive proteins in tomato roots using iTRAQ-based analysis. Biochemical and Biophysical Research Communications, 446(1), 417422. DOI:10.1016/j.bbrc.2014.03.005.

Groppa M D , Benavides M P .2008. Polyamines and abiotic stress: recent advances[J]. Amino Acids 34(1):35-45 DOI: 10.1007/s00726-007-0501-8.

Guo R, Yang Z Z, Li F, Yan C R, Zhong X L, Liu Q, Xia X, Li H R, Zhao L. 2015. Comparative metabolic responses and adaptive strategies of wheat (Triticum aestivum)

PeerJ reviewing PDF | (2021:06:62808:2:0:NEW 19 Jan 2022) 
667

668

669

670

671

672

673

674

675

676

677

678

679

680

681

682

683

684

685

686

687

688

689

690

691

692

693

694

695

696

697

698

699

700

701

702

to salt and alkali stress.BMC Plant Biology 15 (70) :1-13 DOI: 10.1186/s12870-015-0546-X.

Gupta B,Huang B.2014. Mechanism of Salinity Tolerance in Plants: Physiological, Biochemical, and Molecular Characterization.International Journal of Genomics1-18DOI: $10.1155 / 2014 / 701596$.

Gupta J, Downer R G H. 1993. Partial characterization of protein kinase C from an insect cell line. Biochimica et Biophysica Acta (BBA) - Protein Structure and Molecular Enzymology, 1203(2), 210-214. DOI:10.1016/0167-4838(93)90085-6.

Gurmani A R, Bano A, Ullah N,Khan H, Jahangir M,Flowers T J.2013.Exogenous abscisic acid (ABA) and silicon ( $\mathrm{Si})$ promote salinity tolerance by reducing sodium $(\mathrm{Na}+)$ transport and bypass flow in rice (Oryza sativa indica). Australian Journal of Crop Science 7 (9) : 1219-1226

Hajiboland R, Aliasgharzadeh N, Laiegh SF, Poschenrieder C. 2010.Colonization with arbuscular mycorrhizal fungi improves salinity tolerance of tomato (Solanum lycopersicum L.) plants. Plant \& Soil 331(1-2):313-327 DOI: 10.1007/s11104-009-0255-Z.

Hasegawa, M., Bressan, R., \& Pardo, J. M. 2000. The dawn of plant salt tolerance genetics. Trends in Plant Science, 5(8), 317-319. DOI:10.1016/s1360-1385(00)01692-7.

Hazman M, Hause B, Eiche E, Nick P, Riemann M. 2015.Increased tolerance to salt stress in OPDA-deficient rice ALLENE OXIDE CYCLASE mutants is linked to an increased ROSscavenging activity. Journal of Experimental Botany 66(11):3339-3352 DOI: 10.1093/jxb/erv142.

Huang G T, Ma S L, Bai L P, Zhang L, Ma H, Jia P, Liu J, Zhong M, Guo Z F. 2012.Signal transduction during cold, salt, and drought stresses in plants.Molecular Biolog Reports 39(2):969-987DOI: 10.1007/s11033-011-0823-1.

Huaxun Ye, Lei Li, Yanhai Yin.2011.Recent Advances in the Regulation of Brassinosteroid Signaling and Biosynthesis Pathways.Journal of Integrative Plant Biology 53(6): 455-468 DOI: 10.1111/j.1744-7909.2011.01046.x.

Islam F, Farooq M A, Gill R A, Wang J, Yang C,Ali B, Zhou W.2017.2, 4-D attenuates salinity-induced toxicity by mediating anatomical changes, antioxidant capacity and cation transporters in the roots of rice cultivars.Scientific Reports 7 (1) :10443 DOI: 10.1038/s41598-017-09708-X.

Jiang C Q, Cui Q R, Feng K, Xu D F, Li C F, Zheng Q S. 2016. Melatonin improves antioxidant capacity and ion homeostasis and enhances salt tolerance in maize seedlings.Acta Physiologiae Plantarum 38 (4) :82 DOI: 10.1007/s 11738-016-2101-2.

Jian-Kang Zhu,2002. SALT AND DROUGHT STRESS SIGNAL TRANSDUCTION IN PLANTS, Annu. Rev. Plant Biol. 53:247-273DOI: 10.1146/annurev.arplant.53.091401.143329. 
703

704

705

706

707

708

709

710

711

712

713

714

715

716

717

718

719

720

721

722

723

724

725

726

727

728

729

730

731

732

733

734

735

736

737

738

Kanayama Y, Moriguchi R, Deguchi M, Kanahama K, Yamaki S. 2007. Effects of environmental stresses and abscisic acid on sorbitol-6-phosphate dehydrogenase expression in Rosaceae fruit trees. Acta Horticulturae738: 375-381 DOI: 10.17660/actahortic.2007.738.45.

Katalin Gémes, Péter Poór, Edit Horváth, Zsuzsanna Kolbert, Dóra Szopkó, Ágnes Szepesi,Irma Tari.2011.Cross-talk between salicylic acid and $\mathrm{NaCl}$-generated reactive oxygen species and nitric oxide in tomato during acclimation to high salinity.Physiologia Plantarum142(2):179-192DOI: 10.1111/j.1399-3054.2011.01461.x.

Khan H A, Siddique K H M, Colmer T D.2016. Vegetative and reproductive growth of saltstressed chickpea are carbon-limited:sucrose infusion at the reproductive stage improves salt tolerance.Journal of Experimental Botany 68(8): 2001-2011. DOI: 10.1093/jxb/erw177.

LI J, XU H H, LIU W C, ZHANG X W, LU Y T.2015. Ethylene inhibits root elongation during alkaline stress through AUX1 and associated changes in auxin accumulation.Plant Physiology 168(4):1777-1791 DOI: 10.1104/pp.15.00523.

Li M F,Guo $\mathbf{S}$ J, Yang $X$ H, Meng $Q$ W, Wei $X$ J. 2016.Exogenous gammaaminobutyric acid increases salt tolerance of wheat by improving photosynthesis and enhancing activities of antioxidant enzymes. Biol Plant60(1): 123-131 DOI: $10.1093 / j x b / e r w 407$.

LI Q, YANG A, ZHANG W H.2016. Efficient acquisition of iron confers greater tolerance to saline-alkaline stress in rice (Oryza sativaL.).Journal of Experimental Botany67 (22) :64316444. DOI: 10.1093/jxb/erw407.

LI Y Y, XIAO W, WEI X J, GUO S J. 2010.Effects of gamma-aminobutyric acid (GABA) on NaCL-stress of wheat seedlings. Journal of Gansu Agricultural university. 45(02):53-57.

Liming Xiong, Karen S, Schumaker, Jian-Kang Zhu.2002. Supplement: SignalTransduction || Cell Signaling during Cold, Drought, and Salt Stress. The Plant Cell volume 14 DOI: $10.2307 / 3871756$.

Liu A, Yu Y, Duan X, Sun X, Duanmu H, Zhu Y. 2015. GsSKP21, a Glycine soja S-phase kinase-associated protein, mediates the regulation of plant alkaline tolerance and ABA sensitivity.Plant Molecular Biology 87 (1-2) :111-124 DOI: 10.1007/s11103-0140264-Z.

Liu J, Cai H, Liu Y, Zhu Y M, Ji W, Bai X. 2015. A study on physiological characteristics and comparison of salt resistance of two Medicago sativa at the seedling stage.Acta Prataculturae Sinica22 (2) :250-256.

Lovato MB, Martins PS, Lemos Filho JPD. 1994. Germination in Stylosanthes humilis Populations in the Presence of $\mathrm{NaCl}$. Australian Journal of Botany, 42(6), 717. DOI:10.1071/bt9940717. 
739 Lu N W, Duan B L, Li C Y. 2007.Physiological responses to drought and enhanced UV-B

740

741

742

743

744

745

746

747

748

749

750

751

752

753

754

755

756

757

758

759

760

761

762

763

764

765

766

767

768

769

770

771

772

773

774 radiation in two contrasting.Picea asperata populations 37 (7) :1253-1262 DOI: 10.1139/x07-006.

LUO H Y, YANG L W, GAO H B, WU X L,LIU H H .2012.Physiological mechanism of GABA soaking to tomato seed germination and seedling development under $\mathrm{NaCl}$ stress. Acta Bot. Boreal Occident. Sin. 31(11): 2235-2242.

Lutts S, Majerus V, Kinet $\mathbf{J}$ M.1999. $\mathrm{NaCl}$ effects on proline metabolism in rice (Oryza sativa)seedlings.Physiol Plantarum 105(3): 450-458DOI: $\quad 10.1034 / j .1399-$ 3054.1999.105309.x.

M Almansouri, J M Kinet, S Lutts. 2001.Effect of salt and osmotic stresses on germination in durum wheat (Triticum durum Desf.). Plant and Soil 231 (2): 243-254 DOI: 10.1023/A:1010378409663.

Me X Q, Zhang J, Huang B R.2016.Cytokinin-mitigation of salt-induced leaf senescence in perennial ryegrass involving the activation of antioxidant systems and ionic balance.Environmental and Experimental Botany125:1-11 DOI: 10.1016/j.envexpbot.2016.01.002.

Mitsuya S, Takeoka Y, Miyake H. 2000. Effects of sodium chloride on foliar ultrastructure of sweet potato (Ipomoea batatas Lam.) plantlets grown under light and dark conditions in vitro. Journal of Plant Physiology,157(6), 661-667DOI:10.1016/s0176-1617(00)80009-7.

MLLER M, MUNNÉ-BOSCH S.2015. Ethylene response factors:A key regulatory hub in hormone and stress signaling.Plant Physiology169 (1) :32-41 DOI: 10.1104/pp.15.00677.

MSANNE J, LIN J S, STONE J M, AWADA T.2011.Characterization of abiotic stressresponsive Arabidopsis thaliana $\mathrm{RD} 29 \mathrm{~A}$ and $\mathrm{RD} 29 \mathrm{~B}$ genes and evaluation of transgenes.Planta234 (1) :97-107 DOI: 10.1007/s00425-011-1387-y.

Munns Rana;Tester Mark.2008. Mechanisms of Salinity Tolerance. Annu. Rev. Plant Biol. 2008. 59:651-681 DOI:10.1146/annurev.arplant.59.032607.092911.

Murtaza G, Azooz M M, Murtaza B, et al. Nitrogen-use-efficiency (NUE) in plants under $\mathrm{Na} \mathrm{Cl}$ stress[M]//Salt Stress in Plants. Springer New York, 2013: 415-437.

Manaa, A., Faurobert, M., Valot, B., Bouchet, J.-P., Grasselly, D., Causse, M., \& Ahmed, H. B. 2013. Effect of Salinity and Calcium on Tomato Fruit Proteome. OMICS: A Journal of Integrative Biology, 17(6), 338-352. DOI:10.1089/omi.2012.0108.

Muhammad Ali; Muhammad Kamran; Ghulam Hassan Abbasi; Muhammad Hamzah Saleem; Salman Ahmad; Aasma Parveen; Zaffar Malik; Sobia Afzal; Sunny Ahmar; Khadim Muhammad Dawar; Sardar Ali; Saud Alamri; Manzer H. Siddiqui; Rasheed Akbar; Shah Fahad.2021. Melatonin-Induced Salinity Tolerance by Ameliorating Osmotic and Oxidative Stress in the Seedlings of Two Tomato ( Solanum 
775

776

777

778

779

780

781

782

783

784

785

786

787

788

789

790

791

792

793

794

795

796

797

798

799

800

801

802

803

804

805

806

807

808

809

810

lycopersicum L.) Cultivars. Journal of Plant Growth Regulation 40:2236-2248 DOI: 10.1007/s00344-020-10273-3.

Orthen B, Popp M, Smirnoff N. 1994. Hydroxyl radical scavenging properties of cyclitols. Proceedings of the Royal Society of Edinburgh102:269-272 DOI: $10.1017 /$ S0269727000014226.

Pang Q Y, Zhang A Q, Zang W, Yan X F.2016. Integrated proteomics and metabolomics for dissecting the mechanism of global responses to salt and alkali stress in Suaeda corniculate.Plant and Soil 402(1-2):379-394 DOI: 10.1007/s11104-015-2774-0.

Parvin Khursheda; Hasanuzzaman Mirza; Bhuyan M H M Borhannuddin; Mohsin Sayed Mohammad; Fujita And Masayuki.2019._Quercetin Mediated Salt Tolerance in Tomato through the Enhancement of Plant Antioxidant Defense and Glyoxalase Systems. Plants 8(8) :247 DOI: 10.3390/plants8080247.

Rangani J, Parida A K, Panda A, Kumari A.2016. Coordinated changes in antioxidative enzymes protect the photosynthetic machinery from salinity induced oxidative damage and confer salt tolerance in an extreme Halophyte salvadora persica L.Frontiers in Plant Science7(537):1-18 DOI: 10.3389/fpls.2016.00050.

Rozema Jelte, Flowers Timothy.2008. Ecology.Crops for a salinized world. Science 322( 5907): 1478-1480 DOI: 10.1126/science.1168572.

Schreiner T E, Zozor Y. 1996. Salinity influences photosynthetic characteristics water relations and foliar mineral composition Annona squamosa L. Journal of the American Society for Horticultural Science, 121: 243-248.

Scott M D, Meshnick S R, Eaton J W.1987.Superoxide dismutase-rich bacteria.Paradoxical increase in oxidant toxicity. The Journal of Biological Chemistry262(8):36403645DOI:https://schlr.cnki.net/en/Detail/index/SJPDLAST/SJPD13060100522741.

Senaratna Tissa,McKersie Bryan D, Stinson Robert H.1985.Simulation of Dehydration Injury to Membranes from Soybean Axes by Free Radicals. Plant Physiology77 (2) : 472-474 DOI: 10.1104/PP.77.2.472.

Shan X, Zhou H, Sang T, Shu S, Sun J, Guo S.2016.Effects of Exogenous Spermidine on Carbon and Nitrogen Metabolis $\mathrm{m}$ in Tomato Seedlings under High Temperature.Journal of the American Society for Horticultural Science. American Society for Horticultural Science141(4):381-388 DOI: 10.21273/JASHS.141.4.381.

Shao Q S, Shu S, Du J, Xing W. W, Guo S R, Sun J.2015. Effects of NaCl stress on nitrogen metabolism of cucumber seedlings. Russ J Plant Physiol 62(5): 595-603 DOI: 10.1134/S1021443715050155.

SHI S Q, SHI Z, JIANG Z P,QI L W, SUN X M, LI C X, ZHANG S G. 2010.Effects of exogenous $\mathrm{GABA}$ on gene expression of Caragana intermedia roots under $\mathrm{Na} \mathrm{Cl}$ 
811 stress:regulatory roles for $\mathrm{H}_{2} \mathrm{O}_{2}$ and ethylene production.Plant Cell Environ 33(2):149-162

812

813

814

815

816

817

818

819

820

821

822

823

824

825

826

827

828

829

830

831

832

833

834

835

836

837

838

839

840

841

842

843

844

845 DOI: 10.1111/j.1365-3040.2009.02065.x.

Shigeoka S, Ishikawa T, Tamoi M, Miyagawa Y, Takeda T, Yabuta Y, Yoshimura K. 2002. Regulation and function of ascorbate peroxidase isoenzymes. Journal of Experimental Botany 53(372):1305-1319 DOI: 10.1093/jexbot/53.372.1305.

Shweta Mittal,Nilima Kumari,Vinay Sharma. 2012. Differential response of salt stress on Brassica juncea : Photosynthetic performance, pigment, proline, D1 and antioxidant enzymes Plant Physiology and Biochemistry.54.17-26 DOI: 10.1016/j.plaphy.2012.02.003.

Singh N, Bhatla S C.2016.Nitric oxide and iron modulate heme oxygenase activity as a long distance signaling response to salt stress in sunflower seedling cotyledons.Nitric Oxide 53:54-64 DOI: 10.1016/j.niox.2016.01.003.

Smirnoff N. 1993. The role of active oxygen in the response of plants to water deficit and desiccation. New Phytologist 125:27-58DOI: 10.1111/j.1469-8137.1993.tb03863.x.

Smirnoff N. 2005. Antioxidants and reactive oxygen species in plants. Oxford: Blackwell Publishing DOI: 10.1002/9780470988565.

Tester Mark; Davenport Romola. 2003. $\mathrm{Na}^{+}$tolerance and $\mathrm{Na}^{+}$transport in higher plants. Annals of Botany 91(5): 503-527

Torres-Schumann S, Godoy, Jos A. Pintor-Toro.1992.A probable lipid transfer protein gene is induced by $\mathrm{NaCl}$ in stems of tomato plants. Plant Molecular Biology18(4):749-57 DOI: 10.1007/bf00020016.

Tuna A L,Kaya C,Higgs D, Murillo-Amador B, Aydemir S, Girgin A R. 2008. Silicon improves salinity tolerance in wheat plants. Environmental and Experimental Botany, 62(1), 10-16. DOI:10.1016/j.envexpbot.2007.06.006.

Uday K Divi, Tawhidur Rahman, Priti Krishna. 2010.Brassinosteroid-mediated stress tolerance in Arabidopsis shows interactions with abscisic acid, ethylene and salicylic acid pathways. BMC Plant Biology10(1):151 DOI: 10.1186/1471-2229-10-151.

Ungar, I. A. (1996). Effect of Salinity on Seed Germination, Growth, and Ion Accumulation of Atriplex patula (Chenopodiaceae). American Journal of Botany, 83(5), 604-607. DOI:10.1002/j.1537-2197.1996.tb12745.x.

W Chen, C. Feng, W. Guo, D. Shi, C. Yang. 2011.Comparative effects of osmotic-, salt- and alkali stress on growth, photosynthesis, and osmotic adjustment of cotton plants. Photosynthetica 49(3):417 DOI: 10.1007/s11099-011-0050-y.

WEI X D, CHEN G X, SHI D W,LIU D,TANG J H,LI X.2012.Effects of drought on fluorescence characteristics of photosystem II in leaves of Ginkgo biloba. Acta Ecologica Sinica. 32(23): :7492-7500. 
846 Wang Yunlong; Bian Zhonghua; Pan Tonghua; Cao Kai; Zou Zhirong.2021. Improvement

847

848

849

850

851

852

853

854

855

856

857

858

859

860

861

862

863

864

865

866

867

868

869

870

of tomato salt tolerance by the regulation of photosynthetic performance and antioxidant enzyme capacity under a low red to far-red light ratio. Plant Physiology and Biochemistry 167: 806-815 DOI: 10.1016/J.PLAPHY.2021.09.008.

Xia XJ, Wang YJ, Zhou YH, Tao Y, Mao W H, Shi K, Asami T,Chen Z, Yu J Q.2009.Reactive oxygen species are involved in brassinosteroid induced stress tolerande in cucumber.Plant Physiology 150:801-814 DOI: 10.1104/pp.109.138230.

Xiong Liming, Zhu Jian-Kang. 2002. Salt tolerance.The Arabidopsis BookVolume 1, PP e0048 DOI: 10.1199/tab.0048.

Yang Wei, Liu Xiao-dan, Chi Xiao-juan, Wu Chang-ai, Li Yan-ze, Song Li-li, Liu Xiu-ming, Wang Yan-fang, Wang Fa-wei, Zhang Chuang, Liu Yang, Zong Junmei, Li Hai-yan. 2011b. Dwarf apple MbDREB1 enhances plant tolerance to low temperature, drought, and salt stress via both ABA-dependent and ABA-independent pathways. Planta233:219-229 DOI: 10.1007/s00425-010-1279-6.

Zhang A, Zhang J, Ye N, Cao J, Tan M, Zhang J, Jiang M. 2010.ZmMpk5 is required for the NADPH oxidase-mediated self-propagation of apoplastic $\mathrm{H}_{2} \mathrm{O}_{2}$ in brassinosteroid induced antioxidant defence in leaves of maize.J Exp Bot 61:4399-4411DOI: 10.1093/jxb/erq243.

Zhu Q,Zhang J,Gao X,Tong J, Xiao L, Li W, Zhang H .2010.The Arabidopsis AP2/ERF transcription factor RAP2.6 participates in ABA, salt and osmotic stress responses. Gene 457 (1-2) : 1-12 DOI: 10.1016/j.gene.2010.02.011.

Ziogas V,TanouG,Belghazi M, Diamantidis G,Molassiotis A.2016. Characterization of $\beta$ amino- and $\gamma$-amino butyric acid-induced citrus seeds germination under salinity using nanoLC-MS/MS analysis.Plant Cell Rep36(5):787-789 DOI: 10.1007/s00299-016-2063-2.

Peer) reviewing PDF | (2021:06:62808:2:0:NEW 19 Jan 2022) 
Figure 1

Physiological changes between salt-tolerant(ST) and salt-sensitive(SS) cultivars

Basal content of carbohydrate (A), $\mathrm{H}_{2} \mathrm{O}_{2}$ (B) and total proteins (C) in SS and ST. significantly different at $\mathrm{P}<0.05$. Three biological replicates were performed.
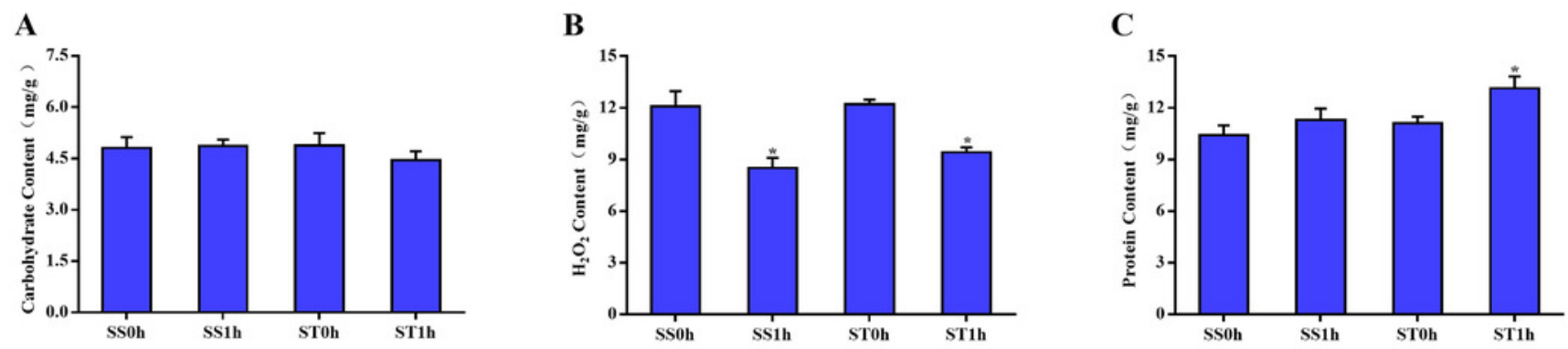


\section{Figure 2}

Landscape of proteomic profiles from all samples

A: Heat map showing the square of Pearson correlation coefficient between samples. The $X$ and $Y$ axes represent each sample. Coloring indicates the square of Pearson correlation coefficient (high: red; low: blue).B: Principle component analysis displayed the separation of all treatments, and stability of replicates with the same treatment. Each circle represents a sample, the circles labeled by same color were the samples with the same treatmentC, D: Two-factor Principle component analysis showing the main variation of proteome distributed in samples after $1 \mathrm{~h}$ imposing, and comparisons of salt-tolerant (ST) and salt-sensitive (SS)cultivars. 
A

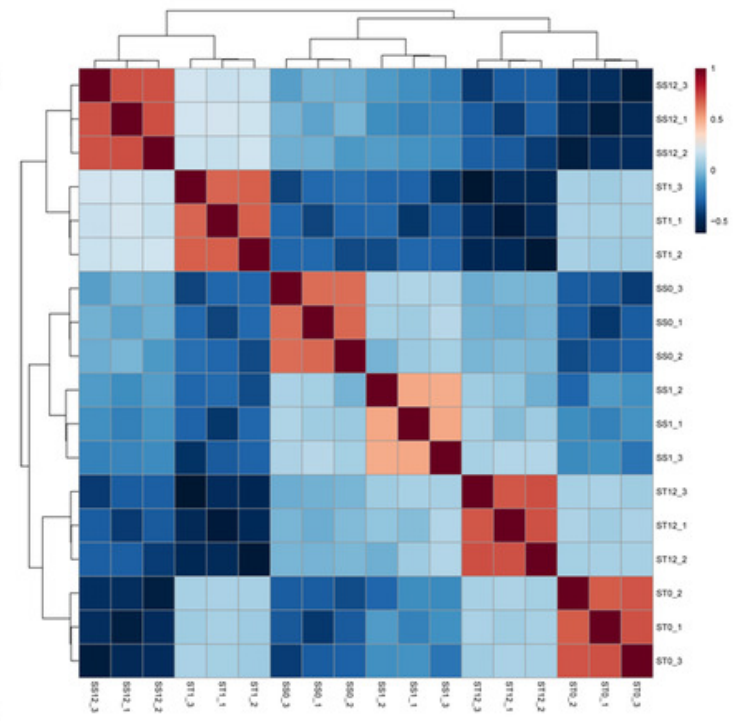

C

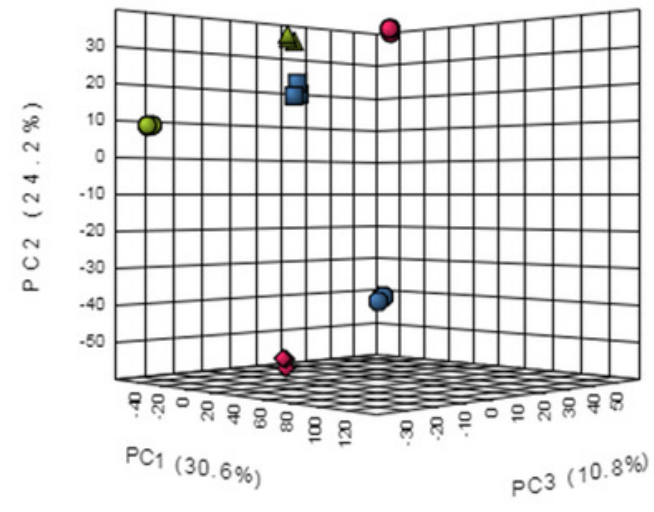

B

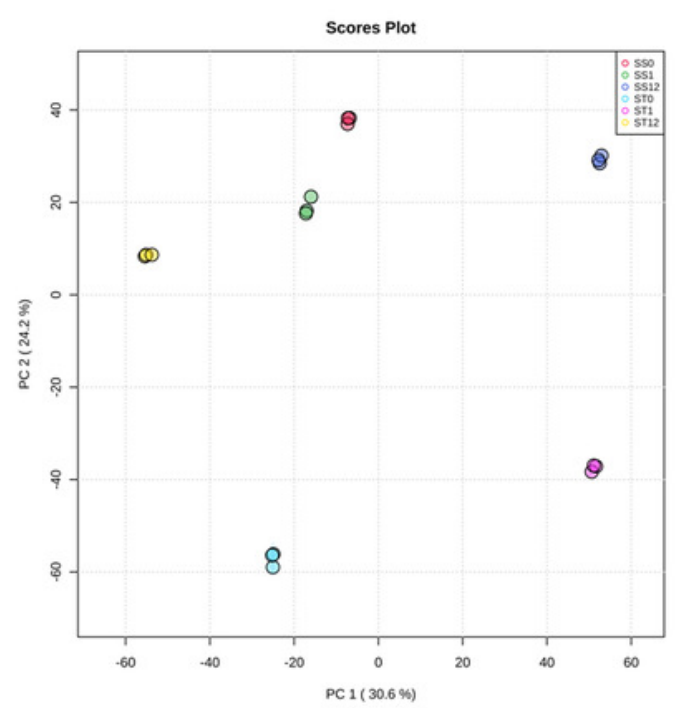

D

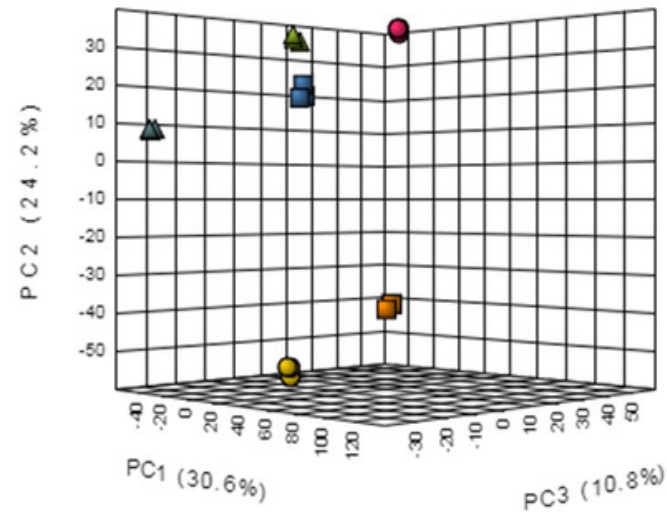

Color Legend

- $\mathrm{sSO}$

- $\$ \$ 11$

- Group 0

- Group 12

Shape Legend

- $\$ 50$

口SS1.

$\Delta S \$ 12$

$\diamond$ STO

O ST1 12 


\section{Figure 3}

Location and variation of differentially expressed proteins between salt-tolerant (ST) and salt-sensitive (SS) cultivars

A: Volcano plots of differentially expressed proteins in SS and ST. Each plots represented a protein identified in SS and ST. The up-regulated proteins (Log1.5(Foldchange) $>1.0 ; \mathrm{P}<$ 0.05 ) were labeled by red, whereas the down-regulated proteins (Log1.5(Foldchange) $<-1.0$; $P<0.05$ ) were shown in blue. B, C, D, E: Basal enzyme activity of Superoxide dismutase (SOD), polyphenol oxidase (PPO), peroxidase (POD) and Polyamine oxidase(PAO)in SS and ST. Asterisk $(*)$ indicates significantlydifferent at $\mathrm{P}<0.05$. Three biological replicates were performed.F: Location of differentially expressed proteins in ST.vs.SS pairwise comparison. 

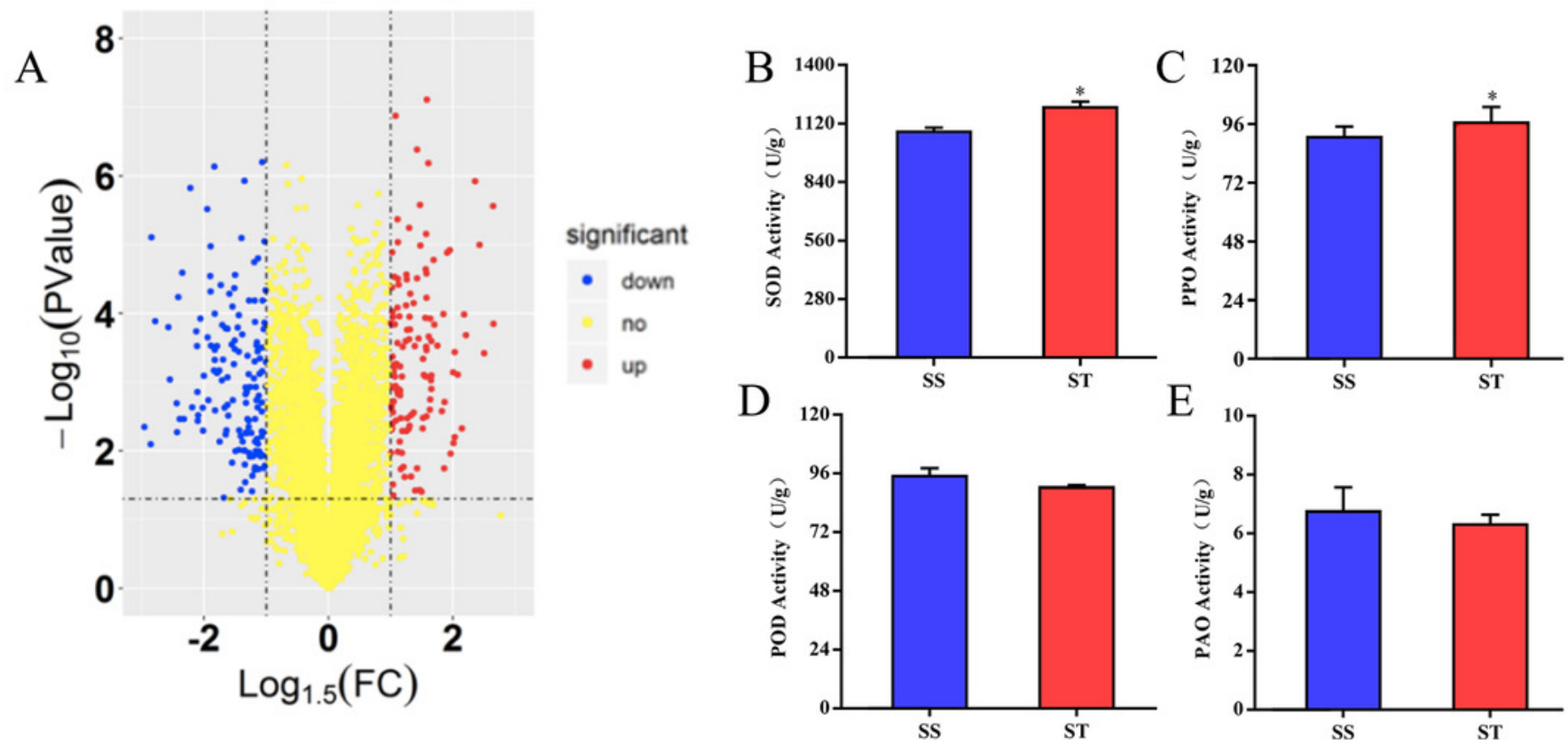

F

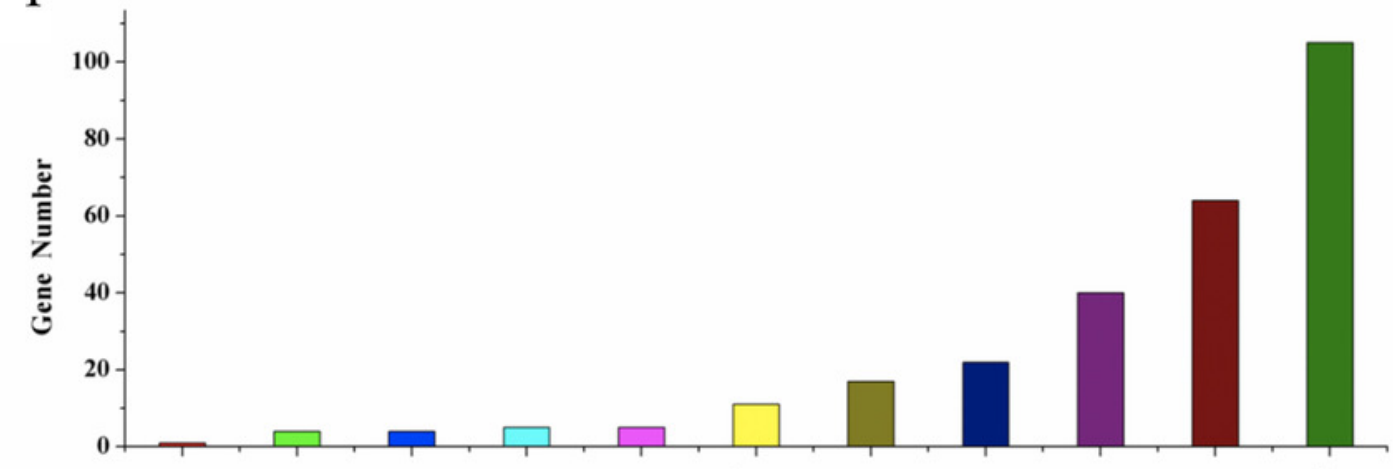

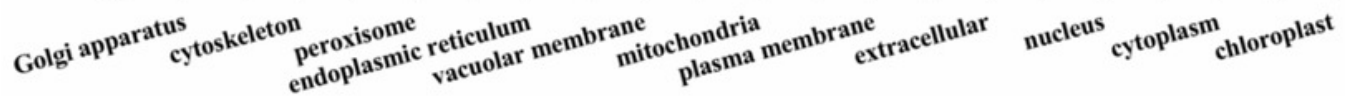

Subcellular Location 


\section{Figure 4}

Function enrichment analysis on all differentially expressed proteins in salt-tolerant (ST) vs salt-sensitive(SS) cultivars

A: All differentially expressed proteins with Gene Ontology matches were assigned to three GO categories (cellular component, molecular function, and biological process). The terms relevant to biological progress was labeled by yellow square, where as the cellular component and molecular function were showed in blue and green squares, respectively. The number of proteins in each term was shown behind the square.B: Sequences with Gene Ontology matches were assigned to the KOG database and classified into 24 functional categories. The number of proteins in each categorizes was shown on the top the each column. 

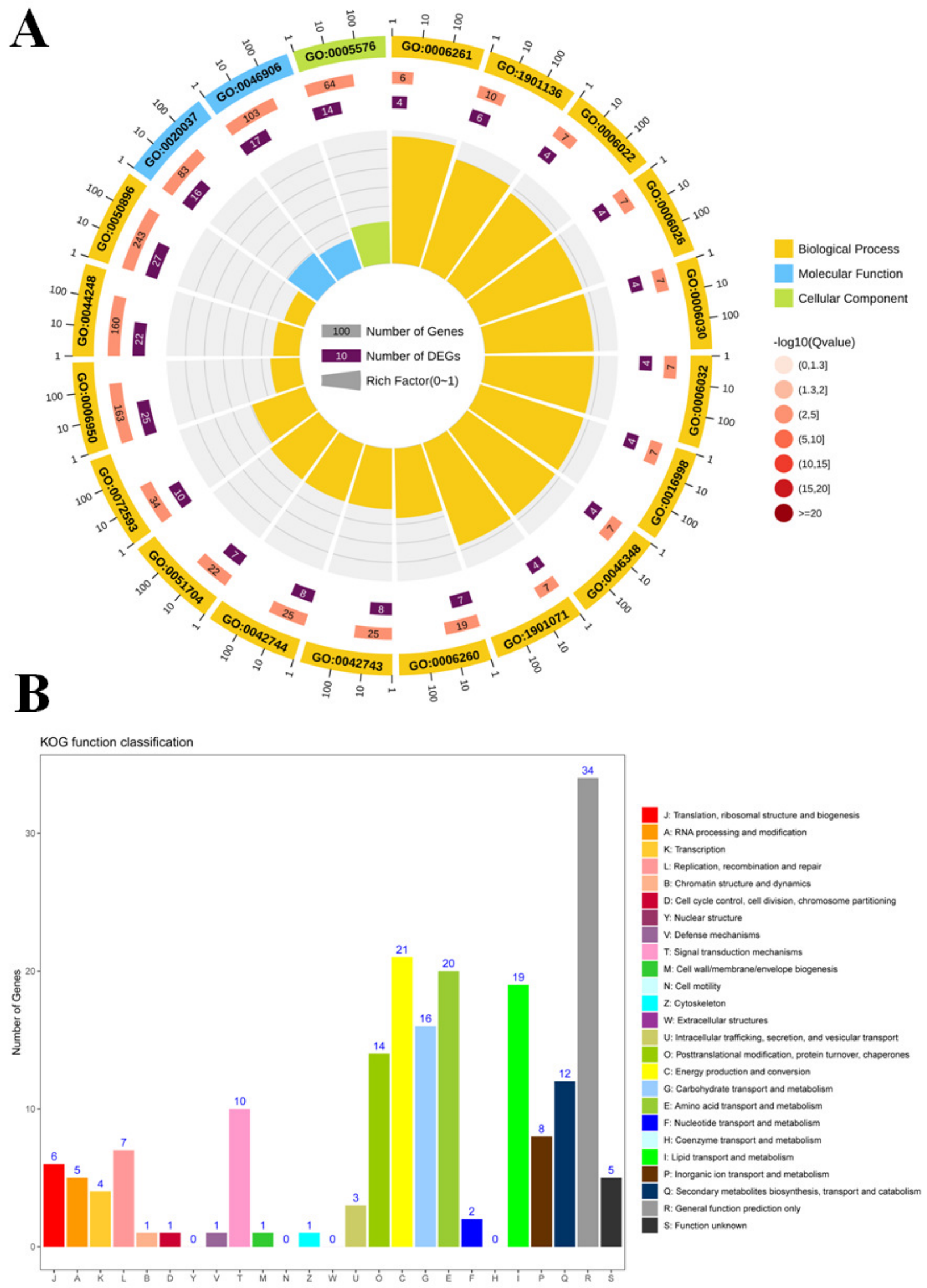


\section{Figure 5}

Pathway enrichment analysis of differentially expressed proteins in salt-tolerant(ST) vs salt-sensitive(SS) cultivars

A: Top 20 of Enriched pathways of differentially expressed proteins; The size of circle represented the number of proteins in the enriched pathway. And the significance were shown in different color (high: blue; low: red; P <0.05)B: Expression pattern of differentially expressed proteins in ST and SS. The highly expressed proteins in ST were shown in red, and the down-regulated proteins in ST were labeled by blue. Each cell represented a differentially expressed protein, and the columns were the samples of SS and ST.

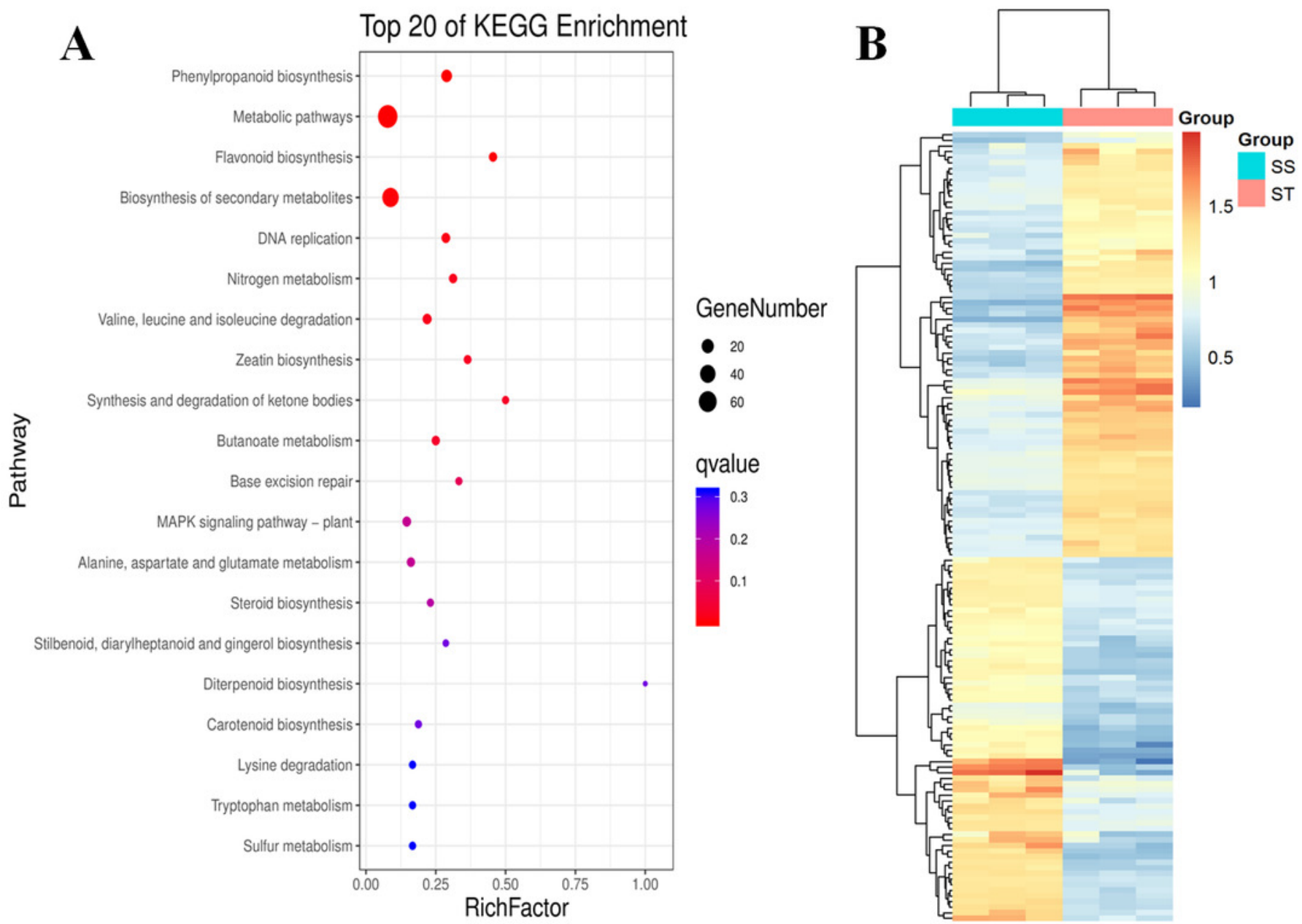




\section{Figure 6}

Location and variation of differentially Expressed proteins between salt-tolerant (ST) and salt-sensitive(SS) cultivars after $1 \mathrm{~h}$ imposing

A: Volcano plots of differentially expressed proteins in SS1 and ST1. Each plots represented a protein identified in SS and ST. The up-regulated proteins (Log1.5(Foldchange) $>1.0 ; \mathrm{P}<$ 0.05) were labeled by blue, whereas the down-regulated proteins (Log1.5(Foldchange) < $-1.0 ; P<0.05)$ were shown in red. The green points were the proteins without significant expression.B: Location of differentially expressed proteins in ST1.vs.SS1 pairwise comparison. Each column represented a structure of organisms.
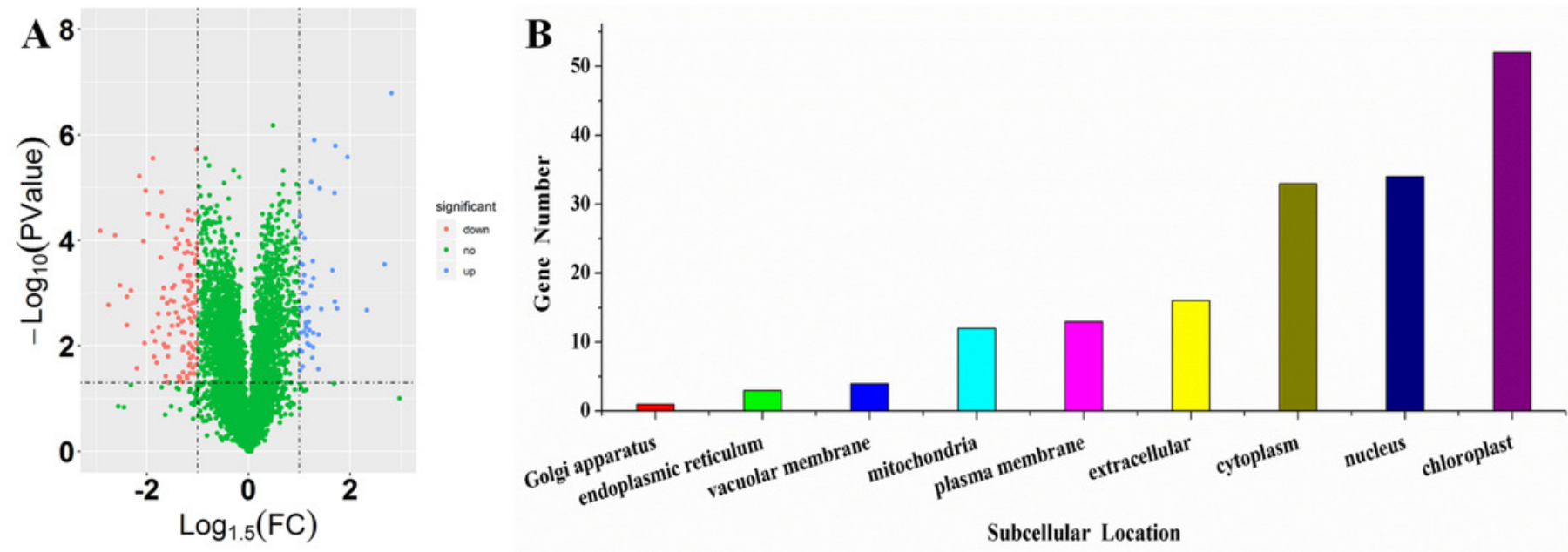


\section{Figure 7}

Function and pathway enrichment analysis on up-regulated proteins in salt-tolerant (ST) after $1 \mathrm{~h}$ imposing

Top 20 of Enriched Gene ontology terms of differentially expressed proteins in ST after 1h imposing; The size of circle represented the number of proteins in the enriched Go terms. And the significance were shown in different color (high: blue; low: red; $\mathrm{P}<0.05$ ); A: Cellular

Component categorize; B: Molecular Function categorize; C: Biological Progress categorize.D: Top 20 of Enriched pathways of differentially expressed proteins in ST following 1h salt stress; The length of colum represented the number of proteins in the enriched pathway. And the significance were shown in different color (high: blue; low: black; P <0.05)E: Expression pattern of differentially expressed proteins in ST and SS following $1 \mathrm{~h}$ salt stress. The highly expressed proteins which involved in response to salt stress in ST were shown in red, and the down-regulated proteins in ST were labeled by green. Each cell represented a differentially expressed protein, and the columns were the samples of SS and ST after 1h imposing. 
A

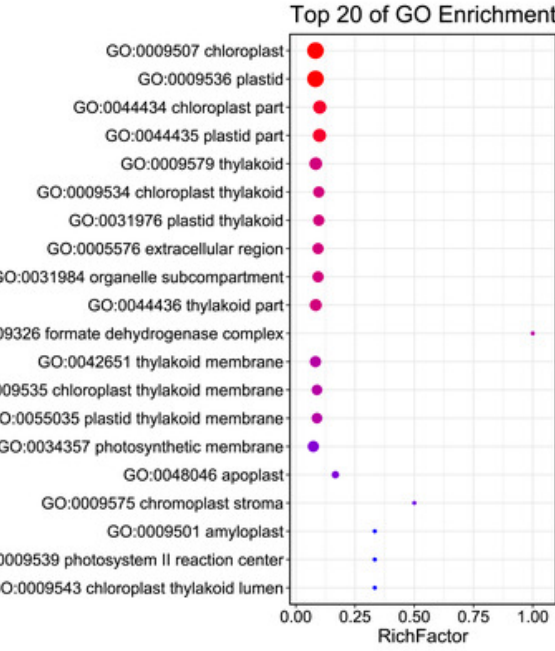

C

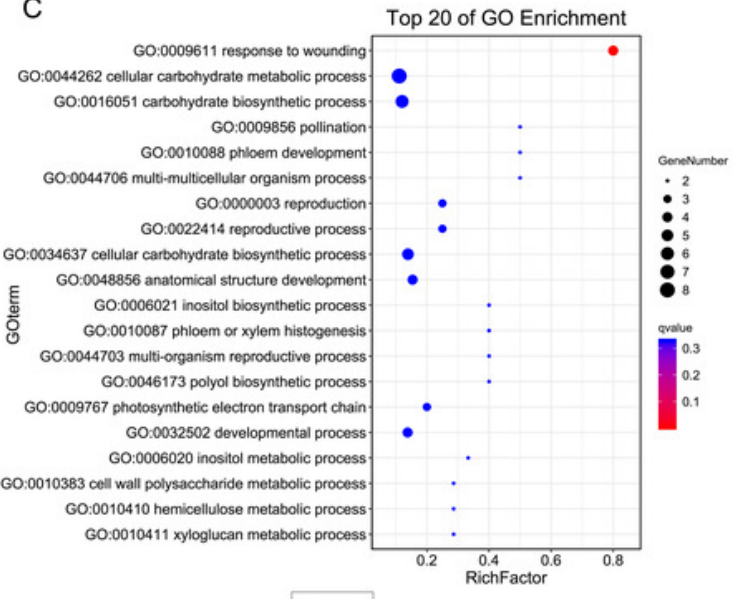

E

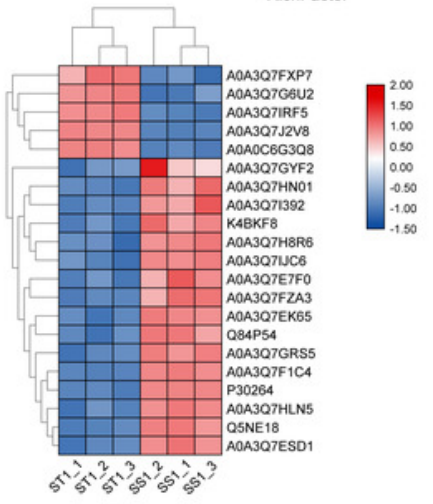

B
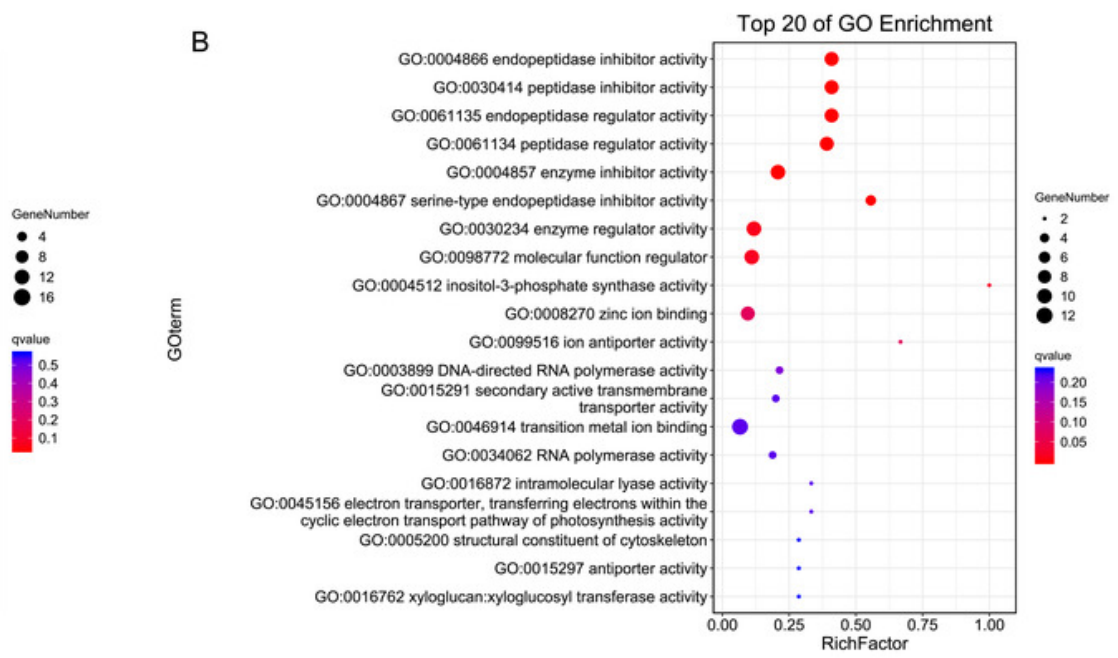

D

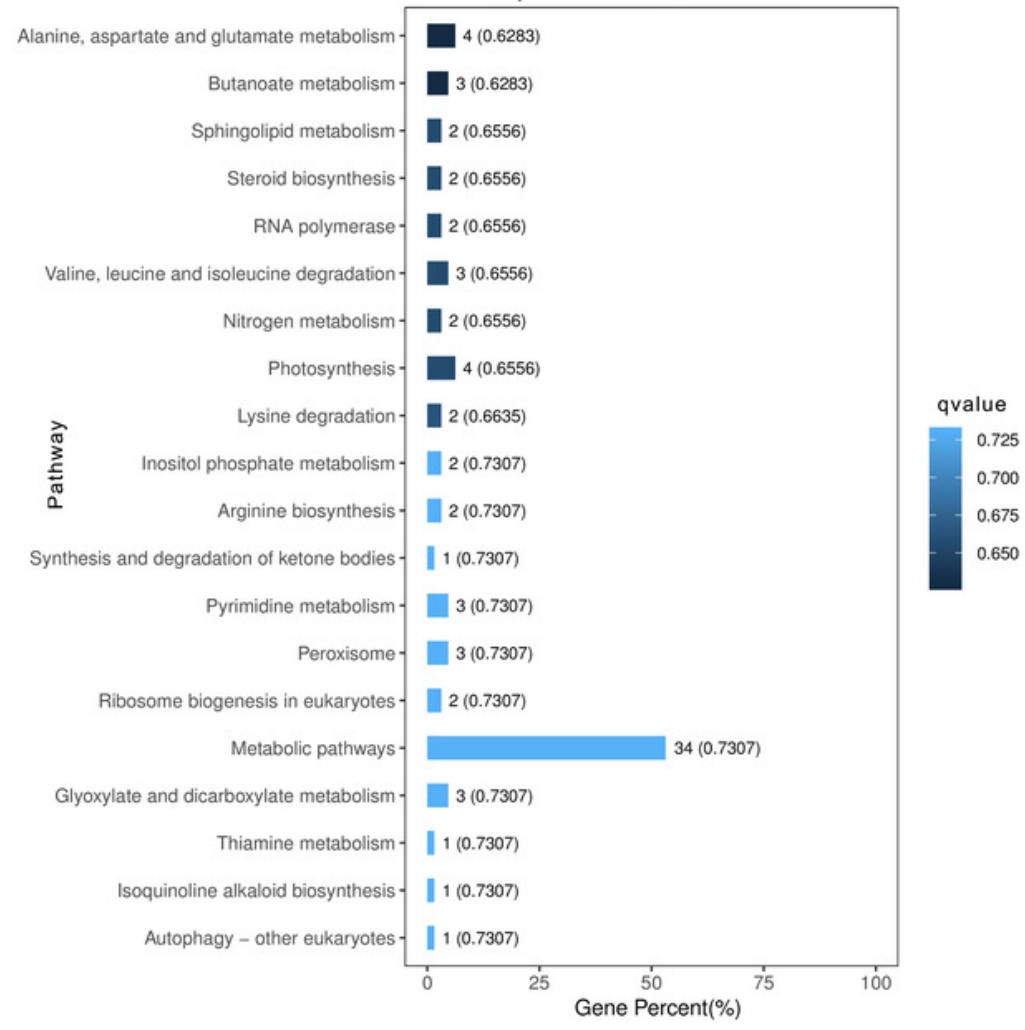




\section{Figure 8}

Three key proteins were involved in mechanism network of resistance of salt-tolerant (ST) to salt stress

A: Venn diagram of up-regulated proteins in ST.vs.SS and ST1.vs.SS1. Three up-regulated proteins were commonly identified in ST and ST1 after $1 \mathrm{~h}$ imposing.B: Co-expression network of all up-regulated proteins which relevant to salt stress in ST. The key genes in the network were shown in red circles, indicating their hub status in response to salt stress. C, D, E:

Relative expression of key proteins in each pairwise comparisons. The column with different color represented a singly pairwise comparison.

A

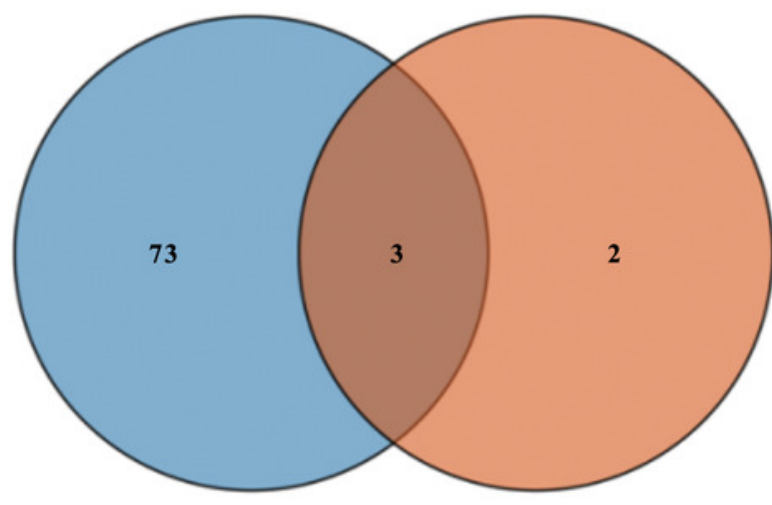

Up-ST.vs.ss

Up-ST1.vs.SS1
B
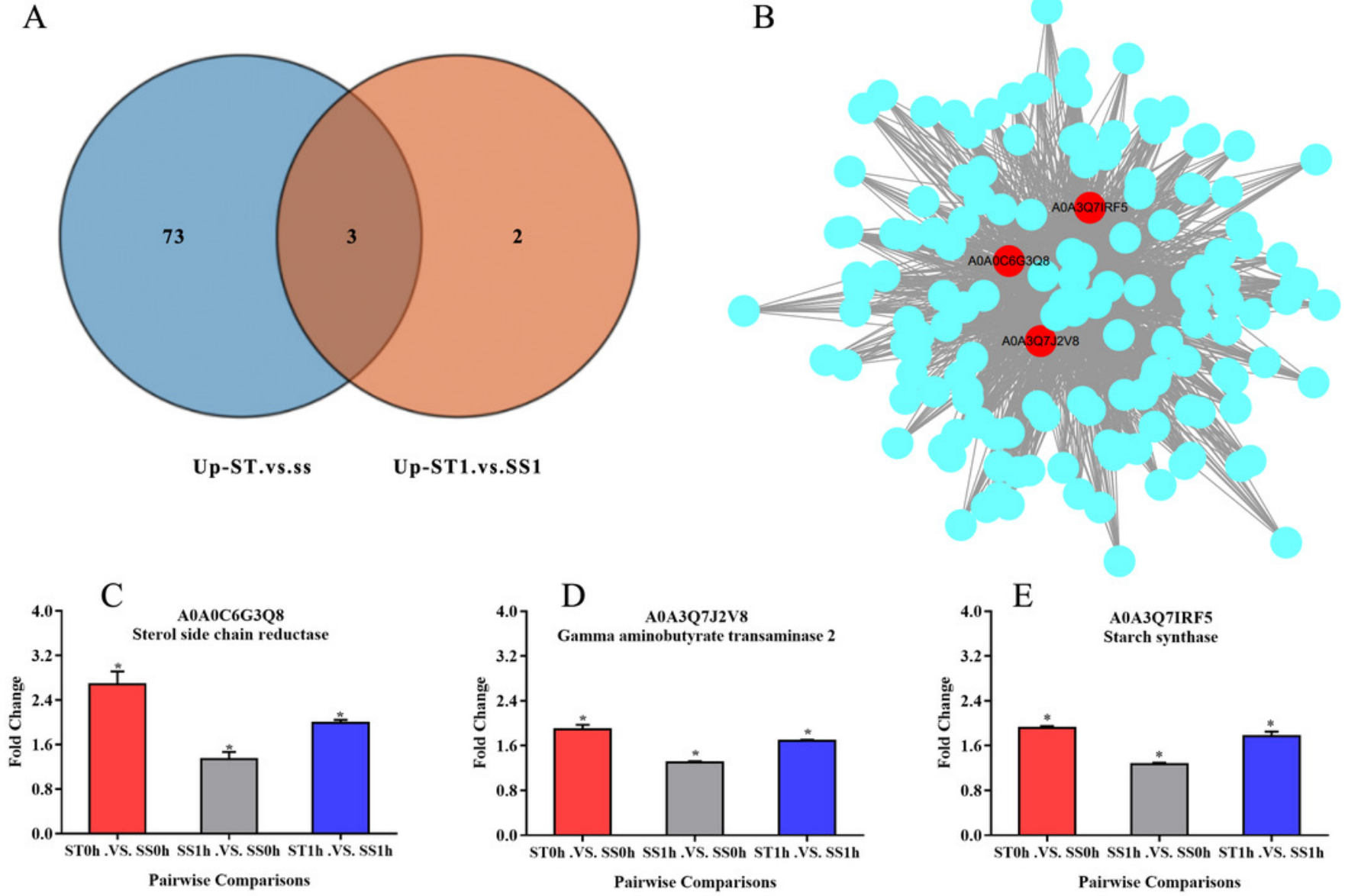

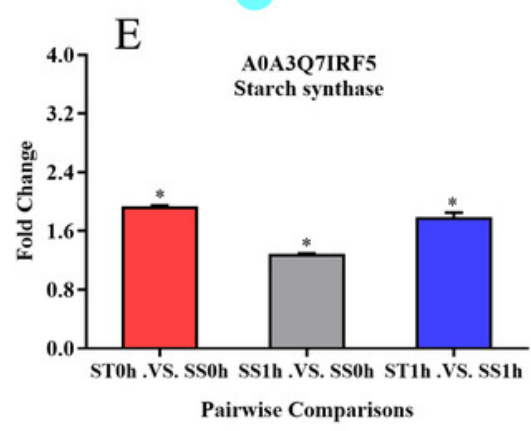


Figure 9

Validation of key genes expression using qRT-PCR

The relative expression level was represented by the $2^{-\Delta \Delta t}$ value, and the SS and ST were shown in green and red columns. Asterisk (**) indicates significantly different at $\mathrm{P}<0.01$.
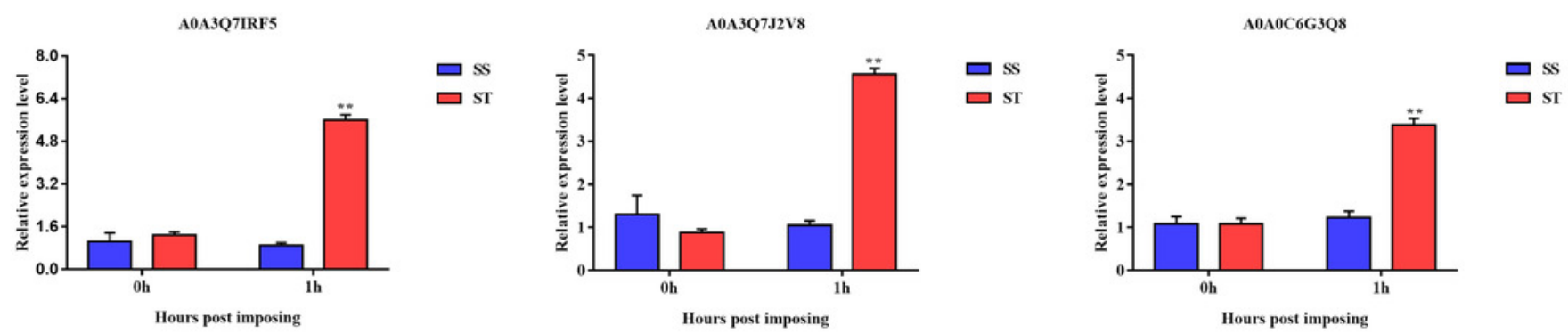


\section{Table 1 (on next page)}

Enzyme activity of salt-tolerant (ST)and salt-sensitive (SS) cultivars under salt stress challenge

significantly different at $\mathrm{P}<0.05$. Three biological replicates were performed. 
Table 1. Enzyme activity of salt-tolerant (ST)and salt-sensitive (SS) cultivars under salt stress challenge

\begin{tabular}{lllll}
\hline \multicolumn{5}{c}{ Enzyme Activity $(\mathrm{U} / \mathrm{g})$} \\
\hline SS0 & $94.99 \pm 3.15 \mathrm{~b}$ & $6.75 \pm 0.82 \mathrm{~b}$ & $90.53 \pm 4.43 \mathrm{bc}$ & $1080.44 \pm 20.60 \mathrm{c}$ \\
SS1 & $49.65 \pm 1.14 \mathrm{~d}$ & $4.30 \pm 0.34 \mathrm{c}$ & $81.28 \pm 5.33 \mathrm{c}$ & $1045.90 \pm 9.24 \mathrm{c}$ \\
ST0 & $90.22 \pm 1.02 \mathrm{c}$ & $6.30 \pm 0.33 \mathrm{~b}$ & $96.57 \pm 6.41 \mathrm{~b}$ & $1197.42 \pm 28.38 \mathrm{a}$ \\
ST1 & $145.98 \pm 1.77 \mathrm{a}$ & $8.67 \pm 0.79 \mathrm{a}$ & $110.52 \pm 4.65 \mathrm{a}$ & $1117.93 \pm 13.24 \mathrm{~b}$ \\
\hline
\end{tabular}

Note: significantly different at $\mathrm{P}<0.05$. Three biological replicates were performed. 


\section{Table 2 (on next page)}

Key proteins of salt-tolerant(ST) in response to salt stress

$P<0.05$ 
Table 2. Key proteins of salt-tolerant(ST) in response to salt stress

Protein accession Protein description

A0A0C6G3Q8 Sterol side chain reductase

gamma aminobutyrate transaminase 2

Starch synthase, chloroplastic/amyloplastic

A0A3Q7J2V8

A0A3Q7IRF5
Subcellular Localization KEGG

plasma membrane

K09828

chloroplast

K16871

Starch syn

1 